\title{
BIFURCATION ANALYSIS OF KOLMOGOROV FLOWS
}

\author{
Mami Matsuda And Sadao MiYatake
}

(Received June 4, 1998, revised February 25, 2002)

\begin{abstract}
We examine the bifurcation curves of solutions to the Kolmogorov problem and present the exact formula for the second derivatives of their components concerning Reynolds numbers at bifurcation points. Using this formula, we show the supercriticality of these curves in the case where the ratio of periodicities in two directions is close to one. In order to prove this, we construct an inverse matrix of infinite order, whose elements are given by sequences generated by continued fractions. For this purpose, we investigate some fundamental properties of these sequences such as quasi-monotonicity and exponential decay from general viewpoints.
\end{abstract}

In 1959, Kolmogorov proposed the investigation of more exact periodic solutions of Navier-Stokes equations in two-dimensional space subject to an external force ${ }^{t}(\gamma \sin y, 0)$ in order to understand the onset of turbulence (see Obukhov [4]). Then, Meshalkin and Sinai [1] studied a related linearized operator, and constructed its eigenfunctions using continued fractions in an explicit way (see Section 1). They proved that Reynolds numbers at bifurcation points are characteristic values of the linearized problem and paved the way to further research. Subsequently, Iudovich [2] considered the stationary problem:

$$
\left\{\begin{array}{l}
u u_{x}+v u_{y}=-P_{x}+v \Delta u+\gamma \sin y, \quad v>0, \quad \gamma>0, \\
u v_{x}+v v_{y}=-P_{y}+v \Delta v, \\
u_{x}+v_{y}=0,
\end{array}\right.
$$

where the unknowns are the velocity vector $V(x, y)={ }^{t}(u(x, y), v(x, y))$ and the pressure $P(x, y)$. In Kolmogorov's problem, solutions are assumed to be doubly periodic:

$$
\left\{\begin{array}{l}
V(x, y)=V(x+2 \pi / \alpha, y)=V(x, y+2 \pi), \\
P(x, y)=P(x+2 \pi / \alpha, y)=P(x, y+2 \pi), \\
\iint_{D} V(x, y) d x d y=0, \quad \iint_{D} P(x, y) d x d y=0,
\end{array}\right.
$$

where $D=\{(x, y) ;|x|<\pi / \alpha,|y|<\pi\}$.

As is well-known, the properties of flow depend on the Reynolds number defined by $\lambda \equiv \gamma / \nu^{2}$. If we scale the unknowns by $V(x, y)=v \tilde{V}(x, y)={ }^{t}(\nu \tilde{u}(x, y), \nu \tilde{v}(x, y))$, and $P(x, y)=v^{2} \tilde{P}(x, y)$, then the new unknowns satisfy (1) with $v=1$ and $\gamma=\lambda$. It is not difficult to see that $V_{0}={ }^{t}\left(u_{0}, v_{0}\right)={ }^{t}\left(\gamma v^{-1} \sin y, 0\right)$ and $P_{0}=0$ is a solution for any $\lambda>0$, which is called the branch of trivial solutions. Hence we are interested in whether or not there

2000 Mathematics Subject Classification. Primary 35Q30; Secondary 76D06, 37Gxx.

Key words and phrases. Navier-Stokes equations, bifurcation, continued fractions. 
exists a solution of (1) and (2) other than $\left(\lambda,\left(V_{0}, P_{0}\right)\right)$. It is known that if $\alpha \geq 1$, then $\left(V_{0}, P_{0}\right)$ is the only solution for any $\lambda>0$ (see [1]).

To discuss the case $\alpha \in(0,1)$, we assume the symmetry of solutions:

$$
V(x, y)=-V(-x,-y), \quad P(x, y)=P(-x,-y) .
$$

Note that $(V, P)=\left(V_{0}, P_{0}\right)$ satisfies (3). Meshalkin-Sinai [1] and Iudovich [2] considered a number $r$ of critical values for each $\alpha \in(0,1)$, depending on $\alpha$ : $\lambda_{1}<\lambda_{2}<\cdots<\lambda_{r}, \lambda_{k}=$ $\lambda(k \alpha), 1 / \alpha-1 \leq r<1 / \alpha$, where $\lambda(\beta)$ is a strictly monotone increasing function defined for $\beta \in[0,1)$ satisfying $\lim _{\beta \rightarrow 0} \lambda(\beta)=\sqrt{2}$ and $\lim _{\beta \rightarrow 1} \lambda(\beta)=\infty$ (see Subsection 1.3 and Appendix). They then proved that in a neighborhood of $\left(\lambda_{k},\left(V_{0}, P_{0}\right)\right), k=1, \cdots, r$, there exists a sequence of nontrivial solutions $\left\{\left(\mu_{n},\left(V_{0}+U_{n}, P_{0}+Q_{n}\right)\right)\right\}_{n=1}^{\infty}$ of (1)-(3) satisfying $U_{n} \rightarrow 0, Q_{n} \rightarrow 0$ and $\mu_{n} \rightarrow \lambda_{k}$ as $n \rightarrow \infty$ in a suitable topology.

The purpose of this paper is to study the structure of the solution set in a neighborhood of bifurcation points $\left(\lambda_{k},\left(V_{0}, P_{0}\right)\right)$. Based on the above results, we can guess that, for each $k=1, \ldots, r$, there exists a bifurcation curve:

$$
\boldsymbol{\Gamma}=\left\{(\mu(s), U(s)) \in C^{2}\left((-1,1) ; R \times \boldsymbol{H}^{3}\right), U(0)=0, \mu(0)=\lambda_{k}\right\},
$$

satisfying, for each $s \in(-1,1)$,

(a) if $\lambda=\mu(s),\left(V_{0}+U(s), P(s)\right)$ is a solution to the problem (1), (2) and (3),

(b) $U(s) \in \boldsymbol{H}^{3} \equiv H^{3} \times H^{3}$ and $P(s) \in H^{4}$,

where $H^{k}$ means the set of periodic functions $H^{k}((2 \pi / \alpha) \mathbf{Z} \times 2 \pi \mathbf{Z})$. Note that $P(s)$ is uniquely determined by $V_{0}+U(s)$ as we see later in $\left(1^{\prime}\right),\left(2^{\prime}\right)$ and $\left(3^{\prime}\right)$. Applying CrandallRabinowitz's bifurcation theorem in [3], we can verify the existence of these curves, which satisfy $\mu^{\prime}(0)=0$.

In this paper, we will prove the precise formula for the second derivative $\mu^{\prime \prime}(0)$. Moreover, from this formula, using repeatedly Abel's criterion for summability concerning the conditional convergence, we can prove that $\mu^{\prime \prime}(0)$ is positive when $\beta=k \alpha$ is close to 1 . In this case, where $\mu(s)$ is called convex in the sense $\mu^{\prime \prime}(0)>0$, we call the bifurcation curve $\boldsymbol{\Gamma}$ supercritical. For the linear problem, $\mu(s)$ corresponds to an eigenvalue $\lambda_{k}$ which is independent of the size $s$ of the eigenvector. Conversely, some meaningful nonlinear phenomena are expected to satisfy the supercriticality: $\mu^{\prime}(0)=0$ and $\mu^{\prime \prime}(0)>0$. As in [4], many researchers studied, from the viewpoint of applications, various numerical approaches and laboratory works after [2]. Recently, Okamoto and Shōji [5] showed many diagrams of numerical analysis for the above problem, which lead us to conjecture the supercriticality of the bifurcation curves and proceed to the mathematical treatment in this paper. From the viewpoint of mathematical physics, Yamada [6] treated the case where $\alpha$ is close to zero (see [5]). Supercriticality means that, besides the basic flow, two more stationary states appear just after the external force becomes larger than a critical value.

Our results can be stated as follows. 
THEOREM 1. Let $\alpha$ belong to $(0,1)$ and $r \in \mathbf{N}$ satisfy $1 / \alpha-1 \leq r<1 / \alpha$. For each $k \in\{1,2, \ldots, r\}$, there exists a bifurcation curve $\boldsymbol{\Gamma}$ as stated above. No other solution exists in a neighborhood of $\left(\lambda_{k}, V_{0}\right)$ except $\left(\lambda, V_{0}\right)$. Moreover, we can verify $\mu^{\prime}(0)=0$.

THEOREM 2. Suppose that all the conditions in Theorem 1 are satisfied. Then we can obtain the precise formula of the value $\mu^{\prime \prime}(0)$, and verify $\mu^{\prime \prime}(0)>0$ if $\beta=k \alpha$ is close to 1 .

REMARK 1. The formula for $\mu^{\prime \prime}(0)$ is represented by Fourier coefficients of the eigenfunction for the linearized operator and elements of the fundamental matrix given in Section 3 (see also Theorem 4, (13'), (16) and (19)). Formulas (16) and (19), which are described by continued fractions that converge rapidly, give us the exact value of $\mu^{\prime \prime}(0)$, which is continuous in $\beta \in(0,1)$. This implies that numerical analyses relying upon this formula will provide us with the precise information as required.

REMARK 2. In order to prove Theorem 2, we shall verify the following facts, which are important in their own right. Fourier coefficients of each eigenfunction for the linearized operator have an almost monotonic property, which we call quasi-monotonicity (see (33) and (35)). Moreover we show that Fourier coefficients decay exponentially (see (34), (34') and Theorem 5).

\section{Reduction of the problem.}

1.1. Stream function. Since $u_{x}+v_{y}=0$, the stream function $\psi(x, y)$ is introduced by the formula

$$
\psi(x, y) \equiv d+\int_{C} u d y-v d x
$$

where $C$ is an arbitrary piecewise smooth curve from $(0,0)$ to $(x, y)$ and $d$ is a constant so chosen that it holds $\iint_{D} \psi d x d y=0$. From this $\psi,(u, v)$ is reproduced by $\left(\psi_{y},-\psi_{x}\right)$. Then, the problem described by (1), (2) and (3) is equivalent to the problem $\left(1^{\prime}\right),\left(2^{\prime}\right)$ and $\left(3^{\prime}\right)$ (see Proposition A-1 in Appendix):

$$
\begin{gathered}
\left\{\begin{array}{l}
v \Delta^{2} \psi+\gamma \cos y=J(\Delta \psi, \psi), \quad J(f, g) \equiv f_{x} g_{y}-f_{y} g_{x}, \\
-\Delta P=2\left(\psi_{x y}^{2}-\psi_{x x} \psi_{y y}\right) .
\end{array}\right. \\
\left\{\begin{array}{l}
\psi(x, y)=\psi(x+2 \pi / \alpha, y)=\psi(x, y+2 \pi), \\
P(x, y)=P(x+2 \pi / \alpha, y)=P(x, y+2 \pi), \\
\iint_{D} \psi(x, y) d x d y=0, \quad \iint_{D} P(x, y) d x d y=0 .
\end{array}\right.
\end{gathered}
$$

$$
\psi(x, y)=\psi(-x,-y), \quad P(x, y)=P(-x,-y) .
$$

By virtue of this transformation we see that it suffices to solve $\psi$ first. As $\iint_{D} P d x d y=0$, $P$ is determined by the second equation in $\left(1^{\prime}\right)$, because $\iint_{D}\left(\psi_{x y}^{2}-\psi_{x x} \psi_{y y}\right) d x d y=0$ is verified to hold by integration by parts. Thus, corresponding to $V=V_{0}+U$, by putting $\psi=\psi_{0}+\gamma \nu^{-1} \varphi$ and $\psi_{0} \equiv-\gamma \nu^{-1} \cos y$, we see that $\left(1^{\prime}\right),\left(2^{\prime}\right)$ and $\left(3^{\prime}\right)$ are equivalent to the 
following $\left(1^{\prime \prime}\right),\left(2^{\prime \prime}\right)$ and $\left(3^{\prime \prime}\right)$.

$$
\begin{gathered}
\Delta^{2} \varphi-\lambda \sin y(\Delta+I) \varphi_{x}-\lambda J(\Delta \varphi, \varphi)=0 . \\
\varphi(x, y)=\varphi(x+2 \pi / \alpha, y)=\varphi(x, y+2 \pi), \quad \iint_{D} \varphi(x, y) d x d y=0 .
\end{gathered}
$$

$$
\varphi(x, y)=\varphi(-x,-y) .
$$

1.2. Function space. Let us denote the two dimensional flat torus $\mathbf{R}^{2} /((2 \pi / \alpha) \mathbf{Z} \times$ $2 \pi \mathbf{Z}$ ) by $M$. We consider the following function space defined on $M$ :

$$
\left\{\begin{array}{l}
X=\left\{\varphi \in H^{4}(M) ; \iint_{M} \varphi(x, y) d x d y=0, \varphi(x, y)=\varphi(-x,-y)\right\} \\
(\varphi, \psi)_{X} \equiv \iint_{M} \Delta^{2} \varphi \Delta^{2} \psi d x d y .
\end{array}\right.
$$

Taking account of $\left(2^{\prime \prime}\right)$ and $\left(3^{\prime \prime}\right)$, we can expand $\varphi \in X$ by the Fourier series

$$
\varphi=\sum_{m=0}^{\infty} \varphi^{(m)}, \quad \varphi^{(0)}=\sum_{n=1}^{\infty} c_{0, n} \cos n y, \quad \varphi^{(m)}=\sum_{n=-\infty}^{\infty} c_{m, n} \cos (m \alpha x+n y),
$$

where $m \geq 1$ and $c_{0,0}=0$. Corresponding to this representation, as stated in [5], we have an orthogonal decomposition $X=X_{0} \oplus X_{1} \oplus \cdots \oplus X_{m} \oplus \cdots$, where

$$
X_{0}=\left\{\varphi^{(0)} ; \sum_{n=1}^{\infty} n^{8}\left|c_{0, n}\right|^{2}<\infty\right\}, \quad X_{m}=\left\{\varphi^{(m)} ; \sum_{n=-\infty}^{\infty}(|m|+|n|)^{8}\left|c_{m, n}\right|^{2}<\infty\right\}, m \in \mathrm{N} .
$$

We can operate $\Delta^{-2}$ to $\left(1^{\prime \prime}\right)$, since these terms contain no $(0,0)$ factor, and then obtain

$$
\left(I-\lambda \Delta^{-2} \sin y(\Delta+I) \partial_{x}\right) \varphi-\lambda \Delta^{-2} J(\Delta \varphi, \varphi)=0 .
$$

For $\varphi \in X$, we denote this relation by

$$
f(\lambda, \varphi)=(I-\lambda L) \varphi-\lambda Q[\varphi]=0,
$$

defining operators $L$ and $Q$ by

$$
L \varphi \equiv \Delta^{-2} \sin y(\Delta+I) \partial_{x} \varphi, \quad Q[\varphi] \equiv \Delta^{-2} J(\Delta \varphi, \varphi) .
$$

Hereafter, we seek a solution $(\lambda(s), \varphi(s))$ of $\left(1^{\prime \prime \prime}\right)$ depending on the parameter $s \in(-1,1)$, which is a nonlinear perturbation to the pair of characteristic values $\lambda(0)$ and eigenfunction space $\left\{t \psi(x) ; t \in \mathbf{R}, \psi(x)=\lim _{s \rightarrow 0}(\varphi(s) / s)\right\}$ of the linearized operator $L$.

1.3. Linearization. Since $f(\lambda, 0)=0$ holds, $\varphi=0$ is a solution of $f(\lambda, \varphi)=0$ for any $\lambda>0$. Let us now consider the linearized operator of $f(\lambda, \varphi)$ at $\varphi=0$, which we denote by $f_{\varphi}(\lambda, 0) \equiv I-\lambda L \equiv A$. Note that we have $A X_{m} \subset X_{m}$ for any $m \geq 0$. Assume that $\varphi \in X$ satisfies the linearized equation $A \varphi=0$, which is equivalent to

$$
\Delta^{2} \varphi^{(m)}-\lambda \sin y(\Delta+I) \partial_{x} \varphi^{(m)}=0, \quad m=0,1,2, \ldots .
$$


From (4), Fourier coefficients $c_{m, n}$ of $\varphi$ satisfy $c_{0, n}=0, n=0,1,2, \ldots$, and

(4') $a_{m, n} b_{m, n}+b_{m, n-1}-b_{m, n+1}=0, n \in \mathbf{Z}, m \in \mathbf{N}, \sum_{m \in \mathbf{N}, n \in \mathbf{Z}}\left(m^{2} \alpha^{2}+n^{2}\right)^{2} b_{m, n}^{2}<\infty$,

where we put

$$
a_{m, n}=\frac{2\left(m^{2} \alpha^{2}+n^{2}\right)^{2}}{\lambda m \alpha\left(m^{2} \alpha^{2}+n^{2}-1\right)}, \quad b_{m, n}=\left(m^{2} \alpha^{2}+n^{2}-1\right) c_{m, n},
$$

as in [1] and [2]. Now, putting $\beta=m \alpha$, we set

$$
a_{n}=a_{n}(\beta, \lambda)=\frac{2\left(\beta^{2}+n^{2}\right)^{2}}{\lambda \beta\left(\beta^{2}+n^{2}-1\right)}, \quad b_{n}=b_{n}(\beta, \lambda)=\left(\beta^{2}+n^{2}-1\right) c_{\beta / \alpha, n} .
$$

Note that $a_{0}<0$ and $a_{n}>0$ for $n \neq 0$. Then we have that

$$
a_{n} b_{n}+b_{n-1}-b_{n+1}=0, \quad n=0, \pm 1, \pm 2, \ldots, \quad \sum_{n}\left(\beta^{2}+n^{2}\right)^{2} b_{n}^{2}<\infty,
$$

which is also represented as

$\left(6^{\prime}\right) \quad \boldsymbol{A}(\beta, \lambda) \boldsymbol{b}=0, \quad \boldsymbol{A}(\beta, \lambda)=\left(\begin{array}{ccccc}\cdots & \cdots & & & \\ 1 & a_{-1} & -1 & & \\ & 1 & a_{0} & -1 & \\ & & 1 & a_{1} & -1 \\ & & & \cdots & \cdots\end{array}\right), \quad \boldsymbol{b}=\left(\begin{array}{l}\vdots \\ b_{-1} \\ b_{0} \\ b_{1} \\ \vdots\end{array}\right)$,

where $\sum_{n}\left(\beta^{2}+n^{2}\right)^{2} b_{n}^{2}<\infty . \boldsymbol{b} \in \ell^{2}$ is equivalent to $\sum_{n}\left(\beta^{2}+n^{2}\right)^{k} b_{n}^{2}<\infty$ for any $k \in \mathbf{N}$ if $\boldsymbol{b}$ is the solution of $\left(6^{\prime}\right)$, as we see below (see $\left(E_{1}\right)$ ). Concerning the uniqueness and the solvability of (6), we have the following proposition due to [1] and [2].

Proposition 1. If $\beta \geq 1, \boldsymbol{b}=0$ is the only solution in $\ell^{2}$ of $\left(6^{\prime}\right)$. For $0<\beta<1$, $\left(6^{\prime}\right)$ has a nonzero solution $\boldsymbol{b}$ in $\ell^{2}$ if and only if $(\beta, \lambda)=(\beta, \lambda(\beta))$ holds.

Here, we briefly review the proof of this proposition, because we will use a similar method. We rely upon the same continued fractions, replacing $\beta$ by $2 \beta$, to construct the inverse matrix of $\boldsymbol{A}(\beta, \lambda)$. First note that, as in [1] and [2], $b_{n} \neq 0$ holds for any $n \in \mathbf{Z}$. Indeed, if $b_{n}=0$ is zero for some $n$, then the system of equations in (6) are separated into two independent groups. In this case we see that $\lim _{n \rightarrow \infty}\left|b_{n}\right|=\infty$ is equivalent to $b_{n+1} \neq 0$. Therefore we can put $\rho_{n} \equiv b_{n} / b_{n-1}$, and then look for $\left\{\rho_{n}\right\}$, which satisfies

$$
\left\{\begin{array}{l}
a_{n}+\frac{1}{\rho_{n}}-\rho_{n+1}=0, \quad n \in \mathbf{Z}, \\
\rho_{n} \longrightarrow 0, \quad \frac{1}{\rho_{-n}} \longrightarrow 0 \quad \text { as } n \rightarrow+\infty .
\end{array}\right.
$$

Once we fix $\rho_{1}$, then other $\rho_{n}$ are determined uniquely by the equations in (7). 
We now introduce the following notation by using infinite continued fractions for any $n \in \mathbf{Z}$.

$$
\left\{\begin{array}{l}
\rho_{n}^{+}=\rho_{n}^{+}(\beta, \lambda) \equiv \frac{-1}{a_{n}}+\frac{1}{a_{n+1}}+\cdots, \\
\rho_{n}^{-}=\rho_{n}^{-}(\beta, \lambda) \equiv a_{n-1}+\frac{1}{a_{n-2}}+\cdots,
\end{array}\right.
$$

where $a_{n}$ are given in (5). Regarding the convergence of continued fractions, see Lemma A-1 in Appendix. Note that $\rho_{n}^{+}<0$ for $n \geq 1$. For $n>1$, it follows from $a_{n}=a_{-n}$ that $\rho_{n}^{+}(\beta, \lambda) \rho_{-n+1}^{-}(\beta, \lambda)=-1$ for all $n$. Since $\lim _{n \rightarrow \pm \infty} a_{n}=\infty$, it also follows that $\lim _{n \rightarrow+\infty} \rho_{n}^{+}=0$ and $\lim _{n \rightarrow-\infty} 1 / \rho_{n}^{-}=0$. Hence, if we choose $\rho_{1}=\rho_{1}^{+}(\beta, \lambda)$ (resp. $\left.\rho_{1}=\rho_{1}^{-}(\beta, \lambda)\right)$ ), then we have $\rho_{n}=\rho_{n}^{+}(\beta, \lambda)$ (resp. $\rho_{n}=\rho_{n}^{-}(\beta, \lambda)$ ) by equations in (7). Otherwise, it is verified that $\lim _{n \rightarrow+\infty}\left|\rho_{n}\right|=\infty\left(\right.$ resp. $\left.\lim _{n \rightarrow-\infty}\left|\rho_{n}\right|^{-1}=\infty\right)$. We prove this fact by an elementary method in Appendix. Another theoretical approach can be seen in [1] and [8].

Therefore, the solution $\rho_{n}$ to (7) exists if and only if $\rho_{1}^{+}(\beta, \lambda)=\rho_{1}^{-}(\beta, \lambda) \equiv \rho_{1}$. By virtue of $a_{n}=a_{-n}$ and (8), this relation is equivalent to

$$
\rho_{1}^{-}(\beta, \lambda)-\rho_{1}^{+}(\beta, \lambda)=a_{0}+2\left(\frac{1}{a_{1}}+\frac{1}{a_{2}}+\cdots\right)=0 .
$$

In Appendix we give a simple proof of the following facts, (see also [1] and [2]):

(i) For $\beta \geq 1$, equation (9) has no real solution $\lambda$.

(ii) For $\beta \in(0,1)$, (9) has a unique solution $\lambda(\beta)$ which is strictly monotone increasing. Moreover, $\lim _{\beta \rightarrow 0} \lambda(\beta)=\sqrt{2}$ and $\lim _{\beta \rightarrow 1} \lambda(\beta)=\infty$.

Moreover we have

(iii) $\left|\rho_{k}^{+}(\beta, \lambda)\right|<2$ for all $k$ and $\lambda$, if $\beta$ is close to 1.

Note that (iii) follows from $\sup _{1 \leq n} a_{n+1} / a_{n}<4$, if we apply Lemma A-3. If (9) holds, the solution of (7) is given by

$$
\rho_{n}^{+}(\beta, \lambda(\beta))=\rho_{n}^{-}(\beta, \lambda(\beta)) \equiv \rho_{n}(\beta) \equiv \rho_{n} \quad \text { for all } n \in \mathbf{Z},
$$

which satisfy $b_{n}=\rho_{n} b_{n-1},(n>0), b_{n}=\left(1 / \rho_{n+1}\right) b_{n+1},(n<0)$ and $\rho_{n} \rho_{-n+1}=-1$. Then, using these $\rho_{n}$, we can represent elements of a solution of (6) as

$$
b_{n}=b_{n}(\beta)= \begin{cases}\left(\prod_{i=1}^{n} \rho_{i}\right) & \text { for } n>0, \\ 1 & \text { for } n=0, \\ \left(\rho_{n+1} \cdots \rho_{0}\right)^{-1}=(-1)^{n} \prod_{i=1}^{-n} \rho_{i} & \text { for } n<0 .\end{cases}
$$

The kernel of $\boldsymbol{A}(\beta, \lambda(\beta))$ in $\ell^{2}$ is given by

$$
\operatorname{ker} \boldsymbol{A}(\beta, \lambda(\beta))=\left\{s \boldsymbol{b} ; \boldsymbol{b}={ }^{t}\left(\cdots, b_{-n}(\beta) \cdots, b_{n}(\beta), \cdots\right), s \in R\right\} .
$$


Here we remark that we can construct a fundamental matrix $\boldsymbol{G}(\beta, \lambda)$, called the Riemann matrix, if $(\beta, \lambda) \neq(\beta, \lambda(\beta))$, in such a way that $\boldsymbol{A}(\beta, \lambda) \boldsymbol{b}=\boldsymbol{f}$ yields $\boldsymbol{b}=\boldsymbol{G}(\beta, \lambda) \boldsymbol{f}$. In Section 3 we consider the case where $\boldsymbol{A}(\beta, \lambda)$ is replaced by ${ }^{t} \boldsymbol{A}(2 \beta, \lambda(\beta))$.

Now, we fix the given $\alpha \in(0,1)$ and put

$$
\lambda_{k} \equiv \lambda(k \alpha), \quad k \in\{1, \ldots, r ; r \alpha<1 \leq(r+1) \alpha\} .
$$

Note that $\beta$ stands for $k \alpha$ in the previous argument. Since the solution $\varphi$ of $(I-\lambda L) \varphi=0$ is decomposed as in Subsection 1.2, the set of characteristic values of $L$ consists of $\left\{\lambda_{k} ; k=\right.$ $1, \ldots, r\}$. Namely, we can find a solution $\varphi \not \equiv 0$ of $(I-\lambda L) \varphi=0$ if and only if $\lambda$ is equal to one of $\left\{\lambda_{k}, k=1, \ldots, r\right\}$. More precisely, by virtue of $b_{n}=b_{n}(k \alpha)$ in (10), the kernel of $A_{k} \equiv I-\lambda_{k} L$ is expressed as

$$
\operatorname{ker} A_{k}=\left\{t \varphi^{(k)} ; \varphi^{(k)}=\sum_{n=-\infty}^{\infty}\left(k^{2} \alpha^{2}+n^{2}-1\right)^{-1} b_{n}(k \alpha) \cos (k \alpha x+n y), t \in \mathbf{R}\right\},
$$

where $\varphi^{(k)} \in X_{k}$. The components of $\varphi^{(k)}=\sum_{n} \varphi_{n}^{(k)} \cos (k \alpha x+n y)$ are given by

$\left(4^{\prime \prime \prime}\right) \quad \varphi_{n}^{(k)} \equiv\left(k^{2} \alpha^{2}+n^{2}-1\right)^{-1} b_{n}(k \alpha), \quad \varphi_{-n}^{(k)}=(-1)^{n} \varphi_{n}^{(k)}, \quad n \in \mathbf{Z}, k=1, \ldots, r$.

Note that $\varphi_{n}^{(k)}$ was denoted also by $c_{k, n}$. From the representation we have the estimate

$$
\left\{\begin{array}{l}
\left|\rho_{n}(\beta)\right| \leq 1 /\left|a_{n}(\beta, \lambda(\beta))\right| \leq \beta \lambda(\beta) / 2 n^{2}, \quad 1 \leq n, \\
\left|b_{n}(\beta)\right| \leq \prod_{i=1}^{n} 1 /\left|a_{i}(\beta, \lambda(\beta))\right| \leq(\beta \lambda(\beta) / 2)^{n}(1 / n !)^{2}, \quad 1 \leq n .
\end{array}\right.
$$

In the next section, we see that the estimate of the Riemann matrix follows from $\left(E_{1}\right)$.

Here we summarize the framework of our argument.

1) We use a suitable implicit function theorem to show the existence of bifurcation curves and show $\mu^{\prime}(0)=0$ by differentiating them at bifurcation points (Section 2).

2) By taking higher derivatives, we can determine an integral representation of $\mu^{\prime \prime}(0)$. In order to derive a more useful expression of $\mu^{\prime \prime}(0)$, we need to solve $z^{\prime}(0)$ appearing in this formula (Subsection 3.1).

3) $z^{\prime}(0)$ satisfies $A_{k} z^{\prime}(0)=\tilde{w}^{(0)}+\tilde{w}^{(2 k)}$, where $\tilde{w}^{(0)} \in X_{0}$ and $\tilde{w}^{(2 k)} \in X_{2 k}$ (Subsection 3.2). Therefore it is necessary to prove that $A_{k}$ has an inverse operator in $X_{0}$ and $X_{2 k}$ (Subsection 3.3).

4) More precisely, we show a rigorous form of the inverse matrix of $\boldsymbol{A}\left(2 k \alpha, \lambda_{k}\right)$ to obtain an exact expression of $\mu^{\prime \prime}(0)$ (Section 4$)$.

5) We then clarify that the main part of $\mu^{\prime \prime}(0)$ appears as $\beta=k \alpha$ tends to 1 . In order to show that this part is positive, we need to prove some facts concerning continued fractions (Section 5).

2. Existence of bifurcation curves. Here we construct bifurcation curves issuing from each $\left(\lambda_{k}, 0\right)$ and prove Theorem 1 . 
2.1. Bifurcation theorem. We use the following bifurcation theorem stated in a convenient form for our use (see [3] and [7] ).

THEOREM 3 (Crandall-Rabinowitz). Let $f(\lambda, \varphi)$ be a function defined on $\mathbf{R} \times X$ with continuous Fréchet derivative $f_{\varphi \lambda}=-L$. For each $k \in\{1, \ldots, r\}$, let $\lambda=\lambda_{k}$ be a characteristic value of $L$ so that $A_{k}=I-\lambda_{k} L$ has a nonzero solution in $X$. Suppose that $f(\lambda, 0) \equiv 0$ in a neighborhood of $\lambda_{k}$. Moreover we assume that

(i) $\operatorname{dim} \operatorname{ker} A_{k}=1$, that is, $\operatorname{ker} A_{k}=\left\{t \varphi^{(k)} ; \varphi^{(k)} \neq 0, t \in \mathbf{R}\right\}$,

(ii) $\Im A_{k}=\left\{A_{k} x ; x \in X\right\}$ is a closed subspace in $X$ and $\operatorname{codim}\left(\Im A_{k}\right)=1$,

(iii) $L \varphi^{(k)} \notin \Im A_{k}$.

Denoting $\left(\operatorname{ker} A_{k}\right)^{\perp}$ simply by $Z_{k}$, we can find a neighborhood $V_{k}$ of $\left(\lambda_{k}, 0\right)$ in $\mathbf{R} \times X$ and a set of continuous functions of a parameter $s \in(-1,1)$ :

$$
\mu_{k}(s):(-1,1) \rightarrow R, \quad z_{k}(s):(-1,1) \rightarrow Z_{k},
$$

satisfying $\mu_{k}(0)=\lambda_{k}, z_{k}(0)=0$, such that the solutions of $f(\lambda, \varphi)=0$ in $V_{k}$ are given by

$$
\left\{\left(\mu_{k}(s), s \varphi^{(k)}+s z_{k}(s)\right) ;|s|<1\right\} \cup\left\{(\lambda, 0) ;(\lambda, 0) \in V_{k}\right\} .
$$

The assumption (i) has been verified in the previous subsection. We can show (ii) by the well-known Lemma 1 and Proposition 2 below.

LemmA 1. Let $A_{k}^{*}\left(=I-\lambda_{k} L^{*}\right)$ be the conjugate operator of $A_{k}$. Then it holds that

$$
\Im A_{k}=\left(\operatorname{ker} A_{k}^{*}\right)^{\perp} .
$$

PROpOSITION 2. Let $r$ be the integer satisfying $r \alpha<1 \leq(r+1) \alpha$. For $k \in$ $\{1, \ldots, r\}$, it holds that $\operatorname{dim} \operatorname{ker} A_{k}^{*}=1$, that is,

$$
\operatorname{ker} A_{k}^{*}=\left\{t \Phi^{(k)} ; \Phi^{(k)}=\sum_{n=-\infty}^{\infty}\left(k^{2} \alpha^{2}+n^{2}\right)^{-2}(-1)^{n} b_{n} \cos (k \alpha x+n y), \quad t \in R\right\},
$$

where $\left\{b_{n}\right\}$ is given in (10).

(iii) follows from Lemma 1 and the following Proposition 3, since we have $\left(L \varphi^{(k)}, \Phi^{(k)}\right)=\left(\varphi^{(k)}, \Phi^{(k)}\right) \neq 0$.

Proposition 3. For $\varphi^{(k)} \in \operatorname{ker} A_{k}$ and $\Phi^{(k)} \in \operatorname{ker} A_{k}^{*}$ defined by

$$
\left\{\begin{array}{l}
\varphi^{(k)}=\sum_{n}\left(k^{2} \alpha^{2}+n^{2}-1\right)^{-1} b_{n} \cos (k \alpha x+n y), \\
\Phi^{(k)}=\sum_{n}\left(k^{2} \alpha^{2}+n^{2}\right)^{-2}(-1)^{n} b_{n} \cos (k \alpha x+n y),
\end{array}\right.
$$

we have $\left(\varphi^{(k)}, \Phi^{(k)}\right)_{X}<0$.

Since $L$ and $L^{*}$ are compact operators, the proof of Lemma 1 is straightforward. For convenience sake, here we prove Proposition 2 and Proposition 3. 
Proof OF Proposition 2. Let $L^{*}$ be the conjugate operator of $L$, which is given by

$$
L^{*}=-\Delta^{-4}(\Delta+I) \sin y \Delta^{2} \partial_{x},
$$

since, for $\varphi, \Phi \in X$,

$$
\begin{aligned}
(L \varphi, \Phi)_{X} & =\iint_{D} \sin y(\Delta+I) \partial_{x} \varphi \Delta^{2} \Phi d x d y=\iint_{D} \partial_{x} \varphi(\Delta+I)\left(\sin y \Delta^{2} \Phi\right) d x d y \\
& =\iint_{D} \Delta^{2} \varphi(-1) \Delta^{-2}(\Delta+I) \sin y \Delta^{2} \partial_{x} \Phi d x d y \equiv\left(\varphi, L^{*} \Phi\right)_{X} .
\end{aligned}
$$

Suppose $A^{*} \Phi=0$ for $\Phi \in X$. Then it follows that

$$
\Delta^{2} \Phi+\lambda_{k} \Delta^{-2}(\Delta+I) \sin y \Delta^{2} \partial_{x} \Phi=0 .
$$

In a similar way, we now obtain the following relations between Fourier coefficients $c_{m, n}^{\prime}$ of $\Phi: c_{0, n}^{\prime}=0, n=0,1,2, \ldots$, and $\boldsymbol{A}\left(\beta, \lambda_{k}\right) \boldsymbol{b}^{\prime}=0$, where $\beta=m \alpha, \boldsymbol{A}\left(\beta, \lambda_{k}\right)$ is the matrix defined in the previous section and $\boldsymbol{b}^{\prime}$ is the column vector with $n$-th component $b_{n}^{\prime}=$ $b_{\beta / \alpha, n}^{\prime}=\left(\beta^{2}+n^{2}\right)^{2}(-1)^{n} c_{\beta / \alpha, n}^{\prime}$. Therefore, Proposition 2 follows in the same way as that for Proposition 1.

ProOF OF Proposition 3. It holds that

$$
\begin{aligned}
\left(\varphi^{(k)}, \Phi^{(k)}\right)_{X} & =\iint_{D} \Delta^{2} \varphi^{(k)} \Delta^{2} \Phi^{(k)} d x d y \\
& =\frac{2 \pi^{2}}{\alpha} \sum_{n=-\infty}^{\infty}\left(k^{2} \alpha^{2}+n^{2}\right)^{2}\left(k^{2} \alpha^{2}+n^{2}-1\right)^{-1}(-1)^{n} b_{n}^{2} .
\end{aligned}
$$

On the other hand, multiplying $(\Delta+I) \varphi^{(k)}$ to $\Delta^{2} \varphi^{(k)}-\lambda_{k} \sin y(\Delta+I) \partial_{x} \varphi^{(k)}=0$, we have

$$
\iint_{D}(\Delta+I) \varphi^{(k)} \Delta^{2} \varphi^{(k)} d x d y-\lambda_{k} \iint_{D}(\Delta+I) \varphi^{(k)} \sin y(\Delta+I) \partial_{x} \varphi^{(k)} d x d y=0 .
$$

Note that the second term vanishes, since integration by parts yields

$$
-\lambda_{k} \iint_{D}(\Delta+I) \varphi^{(k)} \sin y(\Delta+I) \partial_{x} \varphi^{(k)} d x d y=\lambda_{k} \iint_{D}(\Delta+I) \partial_{x} \varphi^{(k)} \sin y(\Delta+I) \varphi^{(k)} d x d y .
$$

Hence we obtain

$$
\iint_{D}(\Delta+I) \varphi^{(k)} \Delta^{2} \varphi^{(k)} d x d y=0
$$

which implies

$$
\sum_{n=-\infty}^{\infty}\left(k^{2} \alpha^{2}+n^{2}\right)^{2}\left(k^{2} \alpha^{2}+n^{2}-1\right)^{-1} b_{n}^{2}=0
$$


since the left-hand side equals

$$
\begin{aligned}
\iint_{D}\left(\sum_{n}(-1) b_{n} \cos (k \alpha x+n y)\right) \\
\quad \times\left(\sum_{n^{\prime}}\left(k^{2} \alpha^{2}+n^{\prime 2}\right)^{2}\left(k^{2} \alpha^{2}+n^{\prime 2}-1\right)^{-1} b_{n^{\prime}} \cos \left(k \alpha x+n^{\prime} y\right)\right) d x d y \\
=-\frac{2 \pi^{2}}{\alpha} \sum_{n=-\infty}^{\infty}\left(k^{2} \alpha^{2}+n^{2}\right)^{2}\left(k^{2} \alpha^{2}+n^{2}-1\right)^{-1} b_{n}^{2}
\end{aligned}
$$

Proposition 3 directly follows from this relation, since only negative valued terms remain in the expansion of $\left(\varphi^{(k)}, \Phi^{(k)}\right)_{X}$.

2.2. Differentiation of a bifurcation curve. Here we restrict ourselves to the solution of

$$
f(\lambda, \varphi)=(I-\lambda L) \varphi-\lambda Q[\varphi]=A_{k} \varphi-\left(\lambda-\lambda_{k}\right) L \varphi-\lambda Q[\varphi]=0
$$

in a neighborhood $V_{k}$ of $\left(\lambda_{k}, 0\right)$ for any fixed $k$. Each solution $(\lambda, \varphi) \in R \times X$ has the following form with a parameter $s:(\lambda(s), \varphi(s)) \equiv\left(\mu_{k}(s), s \varphi^{(k)}+s z_{k}(s)\right)$ for $|s|<1$, satisfying $\mu_{k}(0)=\lambda_{k}, z_{k}(0)=0$ and $\left(z_{k}(s), \varphi^{(k)}\right)_{X}=0$. Then, we can rewrite $f(\lambda(s), \varphi(s))=$ $\varphi(s)-\mu_{k}(s) L \varphi(s)-\mu_{k}(s) Q[\varphi(s)]=0$ into the following form

$$
A_{k} \varphi(s)=\tilde{\mu}_{k}(s) L \varphi(s)+\mu_{k}(s) Q[\varphi(s)], \quad \tilde{\mu}_{k}(s) \equiv \mu_{k}(s)-\lambda_{k} .
$$

Differentiating (11) by $s$, we have that

$$
A_{k} \varphi^{\prime}(s)=\tilde{\mu}_{k}^{\prime}(s) L \varphi(s)+\tilde{\mu}(s) L \varphi^{\prime}(s)+\mu_{k}^{\prime}(s) Q[\varphi(s)]+\mu_{k}(s)(Q[\varphi(s)])^{\prime} .
$$

The second differentiation of (11) gives

$$
\begin{aligned}
A_{k} \varphi^{\prime \prime}(s)= & \tilde{\mu}_{k}^{\prime \prime}(s) L \varphi(s)+2 \tilde{\mu}_{k}^{\prime}(s) L \varphi^{\prime}(s)+\tilde{\mu}(s) L \varphi^{\prime \prime}(s) \\
& +\mu_{k}^{\prime \prime}(s) Q[\varphi(s)]+2 \mu_{k}^{\prime}(s)(Q[\varphi(s)])^{\prime}+\mu_{k}(s)(Q[\varphi(s)])^{\prime \prime} .
\end{aligned}
$$

Putting $s=0$, we have that $\tilde{\mu}(0)=0, \mu_{k}(0)=\lambda_{k}, \varphi(0)=0, L \varphi(0)=0, Q[\varphi(0)]=0$, $\left.(Q[\varphi(s)])^{\prime}\right|_{s=0}=0$ and $\left.(Q[\varphi(s)])^{\prime \prime}\right|_{s=0}=2 Q\left[\varphi^{(k)}\right]$, from which it follows

$$
A_{k} z_{k}^{\prime}(0)=\mu_{k}^{\prime}(0) L \varphi^{(k)}+\lambda_{k} Q\left[\varphi^{(k)}\right] \text {. }
$$

Taking inner-product with $\Phi^{(k)} \in \operatorname{ker} A_{k}^{*} \subset X_{k}$, we obtain $\mu_{k}^{\prime}(0)=0$, if we take account of $Q\left[\varphi^{(k)}\right] \in X_{0} \cup X_{2 k}$ and Proposition 3. Thus we have Theorem 1.

\section{Some analysis at bifurcation points.}

3.1. Integral representation of $\mu_{k}^{\prime \prime}(0)$. It follows from $\left(11^{\prime}\right)$ that

$$
A_{k} z_{k}^{\prime}(0)=\lambda_{k} Q\left[\varphi^{(k)}\right], \quad Q\left[\varphi^{(k)}\right]=\Delta^{-2} J\left(\Delta \varphi^{(k)}, \varphi^{(k)}\right),
$$

which is equivalent to

(12') $\tilde{A}_{k} z_{k}^{\prime}(0) \equiv \Delta A_{k} z_{k}^{\prime}(0)=\left(\Delta^{2}-\lambda_{k} \sin y(\Delta+I) \partial_{x}\right) z_{k}^{\prime}(0)=\lambda_{k} J\left(\Delta \varphi^{(k)}, \varphi^{(k)}\right) \equiv w$.

Differentiate (11) once more, put $s=0$, and divide by three. Then we have that

$$
A_{k} z_{k}^{\prime \prime}(0)=\mu_{k}^{\prime \prime}(0) L \varphi^{(k)}+2 \lambda_{k} \Delta^{-2}\left[J\left(\Delta z_{k}^{\prime}(0), \varphi^{(k)}\right)+J\left(\Delta \varphi^{(k)}, z_{k}^{\prime}(0)\right)\right] .
$$


Taking inner-product with $\Phi^{(k)}$, we have

$$
\mu_{k}^{\prime \prime}(0)=\frac{-2 \lambda_{k}^{2}}{\left(\varphi^{(k)}, \Phi^{(k)}\right)_{X}} \iint_{D}\left(J\left(\Delta z_{k}^{\prime}(0), \varphi^{(k)}\right)+J\left(\Delta \varphi^{(k)}, z_{k}^{\prime}(0)\right)\right) \Delta^{2} \Phi^{(k)} d x d y .
$$

Now, Proposition 3 implies that $-2 \lambda_{k}^{2} /\left(\varphi^{(k)}, \Phi^{(k)}\right)_{X}>0$. In order to determine the value of $\mu_{k}^{\prime \prime}(0)$, it is necessary to solve $z_{k}^{\prime}(0)$ in the equation (12).

3.2. Equations for $z_{k}^{\prime}(0)$. Let us determine the value $z_{k}^{\prime}(0)$ given in (12). First we see that the right-hand side $w$ of (12) can be written as $w=w^{(0)}+w^{(2 k)}$, where $w^{(0)}$ and $w^{(2 k)}$ belong to $\Delta^{2} X_{0}$ and $\Delta^{2} X_{2 k}$, respectively. Namely, let us note

$$
w \equiv \lambda_{k} J\left(\Delta \varphi^{(k)}, \varphi^{(k)}\right) \equiv w^{(0)}+w^{(2 k)}, \quad w^{(0)} \in \Delta^{2} X_{0}, \quad w^{(2 k)} \in \Delta^{2} X_{2 k} .
$$

The components of $w^{(0)}$ and $w^{(2 k)}$ are written explicitly as follows.

PROPOSITION 4. Put $w^{(0)}={ }^{t} \boldsymbol{w}^{(0)} \boldsymbol{c}(0), w^{(2 k)}={ }^{t} \boldsymbol{w}^{(2 k)} \boldsymbol{c}(2 k \alpha)$, where $\boldsymbol{c}(0)$ and $\boldsymbol{c}(2 k \alpha)$ are column vectors with $n$-th components $\cos n y$ and $\cos (2 k \alpha x+n y)$, respectively. Then we obtain the l-th components of the column vectors $\boldsymbol{w}^{(0)}$ and $\boldsymbol{w}^{(2 k)}$ as

$$
\begin{aligned}
{\left(\boldsymbol{w}^{(0)}\right)_{l}}=\frac{\lambda_{k} k \alpha}{2}{ }^{t} \boldsymbol{\varphi}^{(k)} \boldsymbol{K}_{1}\left(S^{l} \boldsymbol{N}-\boldsymbol{N} S^{l}\right) \boldsymbol{\varphi}^{(k)}, \\
\left(\boldsymbol{w}^{(2 k)}\right)_{l}=\frac{\lambda_{k} k \alpha}{2}{ }^{t} \boldsymbol{\varphi}^{(k)} \boldsymbol{K}_{1}\left(\boldsymbol{N} R S^{l}-R S^{l} \boldsymbol{N}\right) \boldsymbol{\varphi}^{(k)},
\end{aligned}
$$

where $\boldsymbol{\varphi}^{(k)}$ is the column vector with $n$-th component $\left(k^{2} \alpha^{2}+n^{2}-1\right)^{-1} b_{n}$ and $\boldsymbol{K}_{1}, \boldsymbol{N}$ are diagonal matrices with $n$-th diagonal elements

$$
\left(\boldsymbol{K}_{1}\right)_{n}=-\left(k^{2} \alpha^{2}+n^{2}\right) \equiv-k_{n}, \quad(\boldsymbol{N})_{n}=n,
$$

respectively. $S^{l}$ and $R$ are matrices with $(i, j)$ elements as follows:

$$
\left(S^{l}\right)_{i, j} \equiv\left\{\begin{array} { l l } 
{ 1 } & { \text { for } j - i = l , } \\
{ 0 } & { \text { otherwise } , }
\end{array} \quad ( R ) _ { i , j } \equiv \left\{\begin{array}{ll}
1 & \text { for } i+j=0 \\
0 & \text { otherwise }
\end{array}\right.\right.
$$

In order to simplify the result, we prove the following proposition.

Proposition 5. The matrices $S, N$ and $R$ defined in Proposition 4 satisfy the following relations :

(i) $S^{l} N-N S^{l}=l S^{l}$,

(ii) $N R S^{l}-R S^{l} N=(2 \boldsymbol{N}-l \boldsymbol{I}) R S^{l}$.

Now, $\left(\boldsymbol{w}^{(0)}\right)_{l}$ and $\left(\boldsymbol{w}^{(2 k)}\right)_{l}$ in Proposition 4 can be written as

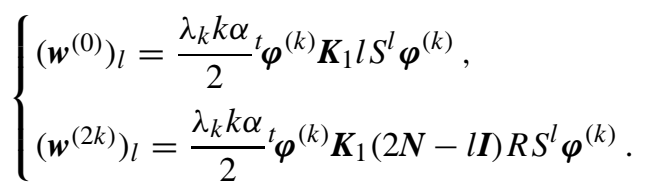

Corresponding to the decomposition $w=w^{(0)}+w^{(2 k)}$, we write the solution of linear equation $\left(12^{\prime}\right)$ as $z^{\prime}{ }_{k}(0)=z^{(0)}+z^{(2 k)}$. Denote $z^{(0)}={ }^{t}{ }^{(0)} \boldsymbol{c}(0)$ and $z^{(2 k)}={ }^{t} z^{(2 k)} \boldsymbol{c}(2 k \alpha)$. Then we see that $\widetilde{A_{k}} z^{(0)}=w^{(0)}$ is equivalent to $z^{t^{(0)}}\left(\widetilde{A_{k}} \boldsymbol{I}\right) \boldsymbol{c}(0)={ }^{t} \boldsymbol{w}^{(0)} \boldsymbol{c}(0)$. Since $\left(\widetilde{A_{k}} \boldsymbol{I}\right) \boldsymbol{c}(0)=\boldsymbol{N}^{4} \boldsymbol{c}(0)$, we 
have ${ }^{t} \boldsymbol{z}^{(0)} \boldsymbol{N}^{4}={ }^{t} \boldsymbol{w}^{(0)}$. From $\left(\boldsymbol{w}^{(0)}\right)_{0}=0$ due to $\left(14^{\prime}\right)$, we can write ${ }^{t} \boldsymbol{z}^{(0)}={ }^{t} \boldsymbol{w}^{(0)} \boldsymbol{N}^{-4}$, where $N^{-1}$ stands for a diagonal matrix with $n$-th element

$$
\left(\boldsymbol{N}^{-1}\right)_{n}= \begin{cases}1 / n & \text { for } n \neq 0 \\ 0 & \text { for } n=0 .\end{cases}
$$

Similarly, $\widetilde{A_{k}} z^{(2 k)}=w^{(2 k)}$ means that ${ }^{t} z^{(2 k)}\left(\widetilde{A_{k}} \boldsymbol{I}\right) \boldsymbol{c}(2 k \alpha)={ }^{t} \boldsymbol{w}^{(2 k)} \boldsymbol{c}(2 k \alpha)$. A direct calculation then gives us $\left(\widetilde{A_{k}} \boldsymbol{I}\right) \boldsymbol{c}(2 k \alpha)=\boldsymbol{\Lambda} \boldsymbol{D} \boldsymbol{c}(2 k \alpha)$, where

$$
\begin{aligned}
\boldsymbol{D} \equiv \boldsymbol{A}\left(2 k \alpha, \lambda_{k}\right) \equiv & \left(\begin{array}{ccccc}
\ddots & \ddots & & & \\
-1 & \tilde{a}_{-1} & 1 & & \\
& -1 & \tilde{a}_{0} & 1 & \\
& & -1 & \tilde{a}_{1} & 1 \\
& & & \ddots & \ddots
\end{array}\right), \quad \boldsymbol{\Lambda} \equiv\left(\begin{array}{ccccc}
\ddots & & & & \\
& \ddot{a}_{-1} & & & \\
& \ddot{a}_{0} & & \\
& & \ddot{a}_{1} & \\
& & & \ddots
\end{array}\right), \\
& \ddot{a}_{n} \equiv \lambda_{k} k \alpha\left(4 k^{2} \alpha^{2}+n^{2}-1\right), \quad \tilde{a}_{n} \equiv a_{n}\left(2 k \alpha, \lambda_{k}\right),
\end{aligned}
$$

$a_{n}(\beta, \lambda)$ being given in (5). Here we have assumed that $k \alpha \neq 1 / 2$. Note that $\tilde{a}_{0}$ is not defined if $k \alpha=1 / 2$. We consider the case where $k \alpha=1 / 2$ later. If $k \alpha \neq 1 / 2$, then we have ${ }^{t} \boldsymbol{z}^{(2 k)} \boldsymbol{\Lambda} \boldsymbol{D}={ }^{t} \boldsymbol{w}^{(2 k)}$.

We now give proofs of Propositions 4 and 5.

Proof of Proposition 4. From the representation of the eigenfunctions of the linearized operator $\varphi^{(k)}={ }^{t} \boldsymbol{\varphi}^{(k)} \boldsymbol{c}(k \alpha)$, it holds that $\Delta \varphi^{(k)}={ }^{t} \boldsymbol{\varphi}^{(k)} \boldsymbol{K}_{1} \boldsymbol{c}(k \alpha)$. Also, $\lambda_{k} J\left(\Delta \varphi^{(k)}, \varphi^{(k)}\right)$ becomes

$$
\begin{aligned}
& \lambda_{k}\left\{\left({ }^{t} \boldsymbol{\varphi}^{(k)} \boldsymbol{K}_{1}(-k \alpha) \boldsymbol{s}(k \alpha)\right)\left({ }^{t} \boldsymbol{\varphi}^{(k)}(-\boldsymbol{N}) \boldsymbol{s}(k \alpha)\right)-\left({ }^{t} \boldsymbol{\varphi}^{(k)} \boldsymbol{K}_{1}(-\boldsymbol{N}) \boldsymbol{s}(k \alpha)\right)\left({ }^{t} \boldsymbol{\varphi}^{(k)}(-k \alpha) \boldsymbol{s}(k \alpha)\right\}\right. \\
& \left.\quad=\lambda_{k} k \alpha^{t} \boldsymbol{\varphi}^{(k)} \boldsymbol{K}_{1}\{\boldsymbol{s}(k \alpha))^{t} \boldsymbol{s}(k \alpha) \boldsymbol{N}-\boldsymbol{N} \boldsymbol{s}(k \alpha){ }^{t} \boldsymbol{s}(k \alpha)\right\} \boldsymbol{\varphi}^{(k)}
\end{aligned}
$$

Note that the $\left(n, n^{\prime}\right)$ element of the matrix $s(k \alpha)^{t} s(k \alpha)$ is given by

$$
\left(s(k \alpha)^{t} s(k \alpha)\right)_{n, n^{\prime}}=\frac{1}{2} \cos \left(n-n^{\prime}\right) y-\frac{1}{2} \cos \left(2 k \alpha x+\left(n+n^{\prime}\right) y\right) .
$$

In general, the matrix $\boldsymbol{M}_{-}$with $\left(n, n^{\prime}\right)$ element $m_{n} n^{\prime}=a\left(n-n^{\prime}\right)$ is given by $\boldsymbol{M}_{-}=$ $\sum a(l) S^{-l}$, and $\boldsymbol{M}_{+}$with $\left(n, n^{\prime}\right)$ element $m_{n} n^{\prime}=b\left(n+n^{\prime}\right)$ equals $\boldsymbol{M}_{+}=\sum b(l) R S^{l}$. Let us write

$\boldsymbol{C}(m) \equiv \sum_{l=-\infty}^{\infty} \cos (m x+l y) S^{-l}, \boldsymbol{C}(m) R \equiv \sum_{l=-\infty}^{\infty} \cos (m x+l y) S^{-l} R=\sum_{l=-\infty}^{\infty} \cos (m x+l y) R S^{l}$,

where we use $R S=S^{-1} R$. In other words, we put

$$
(\boldsymbol{C}(m))_{n, n^{\prime}}=\cos \left(m x+\left(n-n^{\prime}\right) y\right), \quad(\boldsymbol{C}(m) R)_{n, n^{\prime}}=\cos \left(m x+\left(n+n^{\prime}\right) y\right) .
$$


Then $\boldsymbol{s}(k \alpha)^{t} \boldsymbol{s}(k \alpha)$ is equal to $(\boldsymbol{C}(0)-\boldsymbol{C}(2 k \alpha) R) / 2$, and we obtain

$$
\begin{aligned}
\lambda_{k} J\left(\Delta \varphi^{(k)}, \varphi^{(k)}\right)= & \frac{1}{2} \lambda_{k} k \alpha^{t} \boldsymbol{\varphi}^{(k)} \boldsymbol{K}_{1}\{(\boldsymbol{C}(0)-\boldsymbol{C}(2 k \alpha) R) \boldsymbol{N}-\boldsymbol{N}(\boldsymbol{C}(0)-\boldsymbol{C}(2 k \alpha) R)\} \boldsymbol{\varphi}^{(k)} \\
= & \sum_{l=-\infty}^{\infty} \frac{\lambda_{k} k \alpha}{2}{ }^{t} \boldsymbol{\varphi}^{(k)} \boldsymbol{K}_{1}\left(S^{l} \boldsymbol{N}-\boldsymbol{N} S^{l}\right) \boldsymbol{\varphi}^{(k)} \cos l y \\
& +\sum_{l=-\infty}^{\infty} \frac{\lambda_{k} k \alpha}{2} \boldsymbol{\varphi}^{(k)} \boldsymbol{K}_{1}\left(\boldsymbol{N} R S^{l}-R S^{l} \boldsymbol{N}\right) \boldsymbol{\varphi}^{(k)} \cos (2 k \alpha x+l y) .
\end{aligned}
$$

This completes the proof of Proposition 4.

Proof of Proposition 5. If $l=0$, then the both sides of (i) become zero. We use the mathematical induction for $l>0$. If $l=1$, then $S N-N S=S$ follows from a direct calculation. Suppose that $S^{l} N-N S^{l}=l S^{l}$. Then it follows that

$$
\begin{aligned}
S^{l+1} \boldsymbol{N}-\boldsymbol{N} S^{l+1} & =S\left(S^{l} \boldsymbol{N}\right)-\boldsymbol{N} S^{l+1}=S\left(\boldsymbol{N} S^{l}+l S^{l}\right)-\boldsymbol{N} S^{l+1} \\
& =l S^{l+1}+(S \boldsymbol{N}-\boldsymbol{N} S) S^{l}=l S^{l+1}+S^{l+1}=(l+1) S^{l+1} .
\end{aligned}
$$

Thus the identity holds for all $l>0$. For $l<0$, put $l=-l^{\prime}\left(l^{\prime}>0\right)$. The transposition of $S^{l^{\prime}} \boldsymbol{N}-\boldsymbol{N} S^{l^{\prime}}=l^{\prime} S^{l^{\prime}}$ equals $\boldsymbol{N} S^{-l^{\prime}}-S^{-l^{\prime}} \boldsymbol{N}=l^{\prime} S^{-l^{\prime}}$, because ${ }^{t} S=S^{-1}$ and ${ }^{t} S^{l^{\prime}}=S^{-l^{\prime}}$. Hence we obtain (i). As for (ii), first note that $N R=-R N$. Using this relation and (i), we obtain that

$$
N R S^{l}-R S^{l} N=N R S^{l}-R\left(l S^{l}+N S^{l}\right)=(2 N-l \boldsymbol{I}) R S^{l},
$$

which completes the proof of Proposition 5.

3.3. Determination of $z_{k}^{\prime}(0)$ by the fundamental matrix. The inverse matrix of $\boldsymbol{\Lambda}$ is a diagonal matrix with $n$-th element $\left(\boldsymbol{\Lambda}^{-1}\right)_{n}=\ddot{a}_{n}^{-1}$. The existence of the right inverse matrix of $\boldsymbol{D}$, which we call the fundamental matrix, is given as follows.

Proposition 6. Let $k \alpha \neq 1 / 2$. Put $\boldsymbol{D}^{-1}=\left(\cdots \boldsymbol{d}^{(m)} \cdots\right)$, where $\boldsymbol{d}^{(m)}$ are column vectors with $n$-th component $\left(\boldsymbol{d}^{(m)}\right)_{n}=d_{n}^{(m)}$. Then we have that

$$
d_{n}^{(m)}= \begin{cases}\left(\prod_{i=m+1}^{n} \eta_{i}^{+}\right) N_{m+1}^{-1} & \text { for } n>m, \\ N_{m+1}^{-1} & \text { for } n=m \\ \left(\prod_{i=n+1}^{m} \eta_{i}^{-}\right)^{-1} N_{m+1}^{-1} & \text { for } n<m\end{cases}
$$

where

$$
\begin{aligned}
\eta_{n}^{+} & \equiv-\rho_{n}^{+}\left(2 k \alpha, \lambda_{k}\right)=\frac{1}{\tilde{a}_{n}}+\frac{1}{\tilde{a}_{n+1}}+\cdots, \\
\eta_{n}^{-} & \left.\equiv-\rho_{n}^{-}\left(2 k \alpha, \lambda_{k}\right)=-\tilde{a}_{n-1}+\frac{-1}{\tilde{a}_{n-2}}\right\rfloor_{+} \cdots, \quad \tilde{a}_{n}=a_{n}\left(2 k \alpha, \lambda_{k}\right), \\
N_{m+1} & \equiv \eta_{m+1}^{+}-\eta_{m+1}^{-} .
\end{aligned}
$$


Moreover, it holds that

$$
d_{n}^{(m)}=(-1)^{m+n} d_{-n}^{(-m)} \text { for all } n, m \in \mathbf{Z} .
$$

Furthermore, we have another representation of $d_{n}^{(m)}$ for $k \alpha \neq 1 / 2$.

COROLlARY 1. For $k \alpha \neq 1 / 2$, we can represent $d_{n}^{(m)}$ as follows.

(i) For $n \geq 0, m \geq 0$,

$$
d_{n}^{(m)}=(-1)^{m} \prod_{i=1}^{M} \eta_{i}^{+} \prod_{i=1}^{N} \eta_{i}^{-} N_{1}^{-1}
$$

where $M \equiv \max \{|m|,|n|\}, N \equiv \min \{|m|,|n|\}$, and $\prod_{i=1}^{0} \eta_{i}^{ \pm} \equiv 1$.

(ii) For $n \leq 0, m \geq 0$,

$$
d_{n}^{(m)}=(-1)^{m+n} \prod_{i=1}^{m} \eta_{i}^{+} \prod_{i=1}^{|n|} \eta_{i}^{+} N_{1}^{-1} .
$$

Note that we have

$$
N_{m+1}^{-1}=\left(\eta_{m+1}^{+}-\eta_{m+1}^{-}\right)^{-1}=(-1)^{m} \prod_{i=1}^{|m|} \eta_{i}^{+} \eta_{i}^{-} N_{1}^{-1} .
$$

Incidentally, we notice the following slightly general result, whose proof is essentially the same as that of the above proposition.

THEOREM 4. Suppose that $\{a(n)\}_{-\infty}^{\infty}$ satisfies $a(0)>0$ and $c|n|^{2}<a(n), c>0$, for any integer $n \neq 0$. Also, assume that $a(k) \leq a(k+1)\left(1+c_{1} / k^{2}\right)$ and $a(-k) \leq a(-k-$ 1) $\left(1+c_{1} / k^{2}\right)$ for $k \geq 1$. Define $\left\{\rho^{+}(n)\right\}_{-\infty}^{\infty}$ and $\left\{\rho^{-}(n)\right\}_{-\infty}^{\infty}$ respectively by the above $\left\{\eta_{n}^{+}\right\}_{-\infty}^{\infty}$ and $\left\{\eta_{n}^{-}\right\}_{-\infty}^{\infty}$ with $\tilde{a}_{n}$ replaced by $a(n)$. Then the matrix $\boldsymbol{D}$ with $\tilde{a}_{n}$ replaced by $a(n)$ has a right inverse $\boldsymbol{D}^{-1}$. All the components of $\boldsymbol{D}^{-1}$ are expressed by $\left\{\rho_{n}^{+}\right\}_{-\infty}^{\infty}$ and $\left\{\rho_{n}^{-}\right\}_{-\infty}^{\infty}$. Moreover there exists a positive constant $C$ depending on $c$ and $c_{1}$ so that the absolute values of all components $d_{n}^{(m)}$ are uniformly less than $C$.

We give a proof of this theorem in Appendix. Note that it suffices for us to verify the uniform estimate for $d_{n}^{(m)}$. By virtue of this theorem and the estimate $\left(E_{1}\right)$ in Section 1, all series under consideration in this paper are absolutely convergent.

Now, let us return to the problem ${ }^{t}{ }^{(2 k)} \boldsymbol{\Lambda} \boldsymbol{D}={ }^{t} \boldsymbol{w}^{(2 k)}$. By virtue of Proposition 6, we have ${ }^{t} z^{(2 k)}={ }^{t} \boldsymbol{w}^{(2 k)} \boldsymbol{D}^{-1} \boldsymbol{\Lambda}^{-1}$. Thus $z_{k}^{\prime}(0)=z^{(0)}+z^{(2 k)}$ is reprsented by

$$
z_{k}^{\prime}(0)={ }^{t} \boldsymbol{w}^{(0)} \boldsymbol{N}^{-4} \boldsymbol{c}(0)+{ }^{t} \boldsymbol{w}^{(2 k)} \boldsymbol{D}^{-1} \boldsymbol{\Lambda}^{-1} \boldsymbol{c}(2 k \alpha)
$$


We now consider the case of $k \alpha=1 / 2$. Note that $\left(\widetilde{A_{k}} \boldsymbol{I}\right) \boldsymbol{c}(2 k \alpha)=\boldsymbol{D}^{\prime} \boldsymbol{c}(2 k \alpha)$, where

$$
\begin{gathered}
\boldsymbol{D}^{\prime} \equiv\left(\begin{array}{ccccc}
\ddots & \ddots & & & \\
-\ddot{a}_{-1} & \dot{a}_{-1} & \ddot{a}_{-1} & & \\
& -\ddot{a}_{0} & \dot{a}_{0} & \ddot{a}_{0} & \\
& & -\ddot{a}_{1} & \dot{a}_{1} & \ddot{a}_{1} \\
& & & \ddots & \ddots
\end{array}\right), \\
\dot{a}_{n} \equiv\left(4 k^{2} \alpha^{2}+n^{2}\right)^{2}, \quad \ddot{a}_{n} \equiv \lambda_{k} k \alpha\left(4 k^{2} \alpha^{2}+n^{2}-1\right),
\end{gathered}
$$

and that $\dot{a}_{n} / \ddot{a}_{n}$ is equal to $\tilde{a}_{n} \equiv a_{n}\left(2 k \alpha, \lambda_{k}\right)$, if $(n, k \alpha) \neq(0,1 / 2)$. Remark that, if $k \alpha=1 / 2$, $\tilde{a}_{0}$ cannot be defined. However, from

$$
{ }^{t} \boldsymbol{z}^{(2 k)} \boldsymbol{D}^{\prime}={ }^{t} \boldsymbol{w}^{(2 k)}
$$

we can solve ${ }^{t} z^{(2 k)}$, if the right inverse matrix of $\boldsymbol{D}^{\prime}$ exists.

COROLLARY 2. There exists the inverse $\boldsymbol{D}^{\prime-1}=\left(\cdots \boldsymbol{d}^{\prime(m)} \cdots\right)$, where $\boldsymbol{d}^{\prime(m)}$ are column vectors with $n$-th component $\left(\boldsymbol{d}^{\prime(m)}\right)_{n}={d_{n}^{\prime}}^{(m)}$. As for $m=0,1$, we have

$$
d_{n}^{\prime(0)}= \begin{cases}\left(\prod_{i=1}^{n} \eta_{i}^{+}\right) & \text {for } n>0, \\
1 & \text { for } n=0, \quad d_{n}^{\prime(1)}=\left\{\begin{array}{ll}
\left(\prod_{i=2}^{n} \eta_{i}^{+}\right) d^{\prime(1)} & \text { for } n>1, \\
\left(\prod_{i=n+1}^{0} \eta_{i}^{-}\right)^{-1} & \text { for } n<0, \\
0 & \text { for } n=1,
\end{array}, \quad \text { for } n<1,\right.\end{cases}
$$

and for $m \geq 2$

$$
{d^{\prime}}_{n}^{(m)}=\left\{\begin{array}{l}
0 \quad \text { for } n<1, \\
\left\{\left(\dot{a}_{m}+\ddot{a}_{m} \eta_{m+1}^{+}\right)\left(\prod_{i=1}^{m-1} g_{i}\right)-\ddot{a}_{m}\left(\prod_{i=1}^{m-2} g_{i}\right)\right\}^{-1} \quad \text { for } n=1, \\
\left(\prod_{i=1}^{n-1} g_{i}\right) d^{\prime(m)} \quad \text { for } 1<n \leq m \\
\left(\prod_{i=m+1}^{n} \eta_{i}^{+}\right){d^{\prime}}_{m}^{(m)} \quad \text { for } n>m
\end{array}\right.
$$

where

$$
g_{n} \equiv \begin{cases}-\tilde{a}_{n}+\frac{-1}{\tilde{a}_{n-1}}+\cdots+\frac{1}{\tilde{a}_{2}}+\frac{1}{\tilde{a}_{1}} & \text { for } n>1, \\ -\tilde{a}_{1} & \text { for } n=1 .\end{cases}
$$

If we regard $\prod_{i=1}^{0} g_{i} \equiv 1$ and $\prod_{i=1}^{-1} g_{i} \equiv 0$, then the notation for ${d^{\prime}}_{n}^{(m)}$ is also valid for $m=1$. We have similar results when $m<0$ in above proposition. Indeed, it suffices to replace $m, n, \eta_{i}^{+}$and $a_{m}$ by $-m,-n, \eta_{-i+1}^{-}$and $a_{-m}$, respectively.

Now we are in a position to give the proof of Proposition 6. 
ProOF OF Proposition 6. We fix $m$ and solve

$$
\boldsymbol{D d}^{(m)}=\boldsymbol{\delta}_{m},
$$

where $\boldsymbol{\delta}_{m}$ is the column vector whose component is 1 if $n=m$, and 0 otherwise. It then holds that

$$
\tilde{a}_{n} d_{n}^{(m)}-d_{n-1}^{(m)}+d_{n+1}^{(m)}=0 \text { for } n \neq m
$$

Put

$$
\eta_{n}^{(m)} \equiv d_{n}^{(m)} / d_{n-1}^{(m)}
$$

Then $\eta_{n}^{(m)}$ satisfies

$$
\tilde{a}_{n}-\left(\eta_{n}^{(m)}\right)^{-1}+\eta_{n+1}^{(m)}=0 \quad \text { for } n \neq m
$$

As in the proof of Proposition 1, we see that $-\eta_{n}^{(m)}=\rho_{n}^{+}\left(2 k \alpha, \lambda_{k}\right)=-\eta_{n}^{+}$for $m<n$, (resp. $\rho_{n}^{-}\left(2 k \alpha, \lambda_{k}\right)=-\eta_{n}^{-}$for $\left.m>n\right)$, which satisfies

$$
\tilde{a}_{n}+\left(\rho_{n}\right)^{-1}-\rho_{n+1}=0 .
$$

Indeed, if we put $\eta_{m+1}^{(m)}=\eta_{m+1}^{+}$, then it holds that $\eta_{n}^{(m)}=\eta_{n}^{+}$for $n \geq m+1$ and $\lim _{n \rightarrow+\infty} \eta_{n}^{(m)}=0$. For $n \leq m-1$, it also holds, with the notation $\eta_{m}^{(m)}=\eta_{m}^{-}$, that $\eta_{n}^{(m)}=\eta_{n}^{-}$and $\lim _{n \rightarrow-\infty} 1 / \eta_{n}^{(m)}=0$. Note that $\eta_{n}^{+}$are positive and $\eta_{n}^{-}$are negative for all $n$, since all $\tilde{a}_{n}$ are positive in this case. Hence we have $d_{m+1}^{(m)}=\eta_{m+1}^{+} d_{m}^{(m)}, d_{m+2}^{(m)}=$ $\eta_{m+2}^{+} \eta_{m+1}^{+} d_{m}^{(m)}, \ldots$, and

$$
d_{n}^{(m)}=\left(\prod_{i=m+1}^{n} \eta_{i}^{+}\right) d_{m}^{(m)} \text { for } n>m .
$$

Similarly, $d_{m-1}^{(m)}=\left(\eta_{m}^{-}\right)^{-1} d_{m}^{(m)}, d_{m-2}^{(m)}=\left(\eta_{m}^{-} \eta_{m-1}^{-}\right)^{-1} d_{m}^{(m)}, \ldots$, and

$$
d_{n}^{(m)}=\left(\prod_{i=n+1}^{m} \eta_{i}^{-}\right)^{-1} d_{m}^{(m)} \text { for } n<m .
$$

For $n=m$, it holds that $\tilde{a}_{m} d_{m}^{(m)}-d_{m-1}^{(m)}+d_{m+1}^{(m)}=1$, which is equal to

$$
d_{m}^{(m)}\left(\tilde{a}_{m}-\left(\eta_{m}^{(m)}\right)^{-1}+\eta_{m+1}^{(m)}\right)=1 .
$$

Taking account of $\eta_{m}^{(m)}=\eta_{m}^{-}, \eta_{m+1}^{(m)}=\eta_{m+1}^{+}$and $\tilde{a}_{m}-1 / \eta_{m}^{-}=-\eta_{m+1}^{-}$, we obtain

$$
d_{m}^{(m)}\left(\eta_{m+1}^{+}-\eta_{m+1}^{-}\right)=1 .
$$

Denote $N_{m+1} \equiv \eta_{m+1}^{+}-\eta_{m+1}^{-}$. Then we obtain $d_{m}^{(m)}=N_{m+1}^{-1}$. Hence all the other $d_{n}^{(m)}$ can be determined. Now note that it follows $N_{-m+1}=N_{m+1}$ from the above equations and 
$\eta_{i}^{-} \eta_{-i+1}^{+}=-1$. This relation yields for $n>m \in \mathbf{Z}$ that

$$
\begin{aligned}
d_{n}^{(m)} & =\left(\prod_{i=m+1}^{n} \eta_{i}^{+}\right) N_{m+1}^{-1}=\left(\prod_{i=-n+1}^{-m}-\eta_{i}^{-}\right)^{-1} N_{-m+1}^{-1} \\
& =(-1)^{m+n}\left(\prod_{i=-n+1}^{-m} \eta_{i}^{-}\right)^{-1} N_{-m+1}^{-1}=(-1)^{m+n} d_{-n}^{(-m)}
\end{aligned}
$$

This completes the proof of Proposition 6.

4. Precise representation of $\mu_{k}^{\prime \prime}(0)$. As mentioned before, in order to know the sign of $\mu_{k}^{\prime \prime}(0)$, it suffices to consider the sign of the right hand side of (13). Noting $z_{k}^{\prime}(0)=$ $z^{(0)}+z^{(2 k)}$, we rewrite (13) as

$$
\mu_{k}^{\prime \prime}(0)=\frac{1}{p_{k}}\left(D_{1}^{(k)}+D_{2}^{(k)}\right), \quad p_{k} \equiv \frac{\left(\varphi^{(k)}, \Phi^{(k)}\right)_{X}}{-2 \lambda_{k}^{2}}>0,
$$

where $D_{1}^{(k)}$ and $D_{2}^{(k)}$ are given by

$$
\left\{\begin{array}{l}
D_{1}^{(k)} \equiv \iint_{D}\left\{J\left(\Delta z^{(0)}, \varphi^{(k)}\right)+J\left(\Delta \varphi^{(k)}, z^{(0)}\right)\right\} \Delta^{2} \Phi^{(k)} d x d y, \\
D_{2}^{(k)} \equiv \iint_{D}\left\{J\left(\Delta z^{(2 k)}, \varphi^{(k)}\right)+J\left(\Delta \varphi^{(k)}, z^{(2 k)}\right)\right\} \Delta^{2} \Phi^{(k)} d x d y .
\end{array}\right.
$$

In this section we describe the formula for $D_{1}^{(k)}+D_{2}^{(k)}$ more precisely and estimate its value as $\beta$ tends to 1 .

4.1. $\quad D_{1}^{(k)}$ and its positiveness. First, let us describe $D_{1}^{(k)}$ by substituting Fourier expansions of

$$
z^{(0)}={ }^{t} \boldsymbol{w}^{(0)} \boldsymbol{N}^{-4} \boldsymbol{c}(0), \quad \varphi^{(k)}={ }^{t} \boldsymbol{\varphi}^{(k)} \boldsymbol{c}(k \alpha), \quad \Phi^{(k)}={ }^{t} \boldsymbol{\Phi}^{(k)} \boldsymbol{c}(k \alpha),
$$

where $\boldsymbol{w}^{(0)}, \boldsymbol{N}$ and $\boldsymbol{c}(\cdot)$ are given in Proposition 4 , and $\boldsymbol{\varphi}^{(k)}$ and $\boldsymbol{\Phi}^{(k)}$ are column vectors with $n$-th component $\left(k^{2} \alpha^{2}+n^{2}-1\right)^{-1} b_{n}$ and $\left(k^{2} \alpha^{2}+n^{2}\right)^{-2}(-1)^{n} b_{n}$, respectively. Recall that $b_{n}$ is given in (10). Now we have

$$
J\left(\Delta z^{(0)}, \varphi^{(k)}\right)=-\left(-{ }^{t} \boldsymbol{w}^{(0)} \boldsymbol{N}^{-2}(-\boldsymbol{N}) \boldsymbol{s}(0)\right)\left(-k \alpha^{t} \boldsymbol{\varphi}^{(k)} \boldsymbol{s}(k \alpha)\right)=k \alpha^{t} \boldsymbol{w}^{(0)} \boldsymbol{N}^{-1} \boldsymbol{s}(0)^{t} \boldsymbol{s}(k \alpha) \boldsymbol{\varphi}^{(k)} .
$$

Since the $\left(n, n^{\prime}\right)$ element of the matrix $\boldsymbol{s}(0){ }^{t} \boldsymbol{s}(k \alpha)$ is

$$
\begin{aligned}
\left(\boldsymbol{s}(0)^{t} \boldsymbol{s}(k \alpha)\right)_{n, n^{\prime}} & =\sin n y \sin \left(k \alpha x+n^{\prime} y\right) \\
& =\frac{1}{2} \cos \left(-k \alpha x+\left(n-n^{\prime}\right) y\right)-\frac{1}{2} \cos \left(k \alpha x+\left(n+n^{\prime}\right) y\right),
\end{aligned}
$$

it holds that $\boldsymbol{s}(0) \boldsymbol{t}(k \alpha)=(\boldsymbol{C}(-k \alpha)-\boldsymbol{C}(k \alpha) R) / 2$, where $\boldsymbol{C}(-\beta)$ and $\boldsymbol{C}(\beta) R$ are given respectively by $\boldsymbol{C}(-\beta)=\sum_{l=-\infty}^{\infty} \cos (-\beta x+l y) S^{-l}=\sum_{l=-\infty}^{\infty} \cos (\beta x+l y) S^{l}$ and $\boldsymbol{C}(\beta) R=$ 
$\sum_{l=-\infty}^{\infty} \cos (\beta x+l y) R S^{l}$. Note that $S^{-l} R=R S^{l}$. Then it follows that

$$
\begin{aligned}
J\left(\Delta z^{(0)}, \varphi^{(k)}\right) & =k \alpha^{t} \boldsymbol{w}^{(0)} \boldsymbol{N}^{-1} \frac{1}{2}(\boldsymbol{C}(-k \alpha)-\boldsymbol{C}(k \alpha) R) \boldsymbol{\varphi}^{(k)} \\
& =\frac{k \alpha}{2} \boldsymbol{w}^{(0)} \boldsymbol{N}^{-1} \sum_{l=-\infty}^{\infty} \cos (k \alpha x+l y)(I-R) S^{l} \boldsymbol{\varphi}^{(k)} \equiv{ }^{t} \boldsymbol{J}_{1} \boldsymbol{c}(k \alpha),
\end{aligned}
$$

where the $n$-th component of $\boldsymbol{J}_{1}$ is $\left(\boldsymbol{J}_{1}\right)_{n} \equiv(k \alpha) / 2^{t} \boldsymbol{w}^{(0)} \boldsymbol{N}^{-1}(I-R) S^{n} \boldsymbol{\varphi}^{(k)}$. Now it holds

$$
J\left(\Delta z^{(0)}, \varphi^{(k)}\right) \Delta^{2} \Phi^{(k)}={ }^{t} \boldsymbol{J}_{1} \boldsymbol{c}(k \alpha)^{t} \boldsymbol{\Phi}^{(k)} \boldsymbol{K}_{1}^{2} \boldsymbol{c}(k \alpha)={ }^{t} \boldsymbol{J}_{1} \boldsymbol{c}(k \alpha)^{t} \boldsymbol{c}(k \alpha) \boldsymbol{K}_{1}^{2} \boldsymbol{\Phi}^{(k)},
$$

where $\boldsymbol{K}_{1}$ is given in Proposition 4. Since the $\left(n, n^{\prime}\right)$ element of the matrix $\boldsymbol{c}(k \alpha)^{t} \boldsymbol{c}(k \alpha)$ is given by

$$
\begin{aligned}
\left(\boldsymbol{c}(k \alpha)^{t} \boldsymbol{c}(k \alpha)\right)_{n, n^{\prime}} & =\cos (k \alpha x+n y) \cos \left(k \alpha x+n^{\prime} y\right) \\
& =\frac{1}{2} \cos \left(2 k \alpha x+\left(n+n^{\prime}\right) y\right)+\frac{1}{2} \cos \left(n-n^{\prime}\right) y,
\end{aligned}
$$

we have $\boldsymbol{c}(k \alpha)^{t} \boldsymbol{c}(k \alpha)=\left(\boldsymbol{C}^{\prime}(2 k \alpha)+\boldsymbol{C}(0)\right) / 2$ and obtain

$$
\begin{aligned}
J\left(\Delta z^{(0)}, \varphi^{(k)}\right) \Delta^{2} \Phi^{(k)} & =\frac{1}{2}^{t} \boldsymbol{J}_{1}\left(\boldsymbol{C}^{\prime}(2 k \alpha)+\boldsymbol{C}(0)\right) \boldsymbol{K}_{1}^{2} \boldsymbol{\Phi}^{(k)} \\
& =\frac{1}{2}{ }^{t} \boldsymbol{J}_{1} \sum_{l=-\infty}^{\infty} \cos (2 k \alpha x+l y) R S^{l} \boldsymbol{K}_{1}^{2} \boldsymbol{\Phi}^{(k)}+\frac{1}{2}^{t} \boldsymbol{J}_{1} \sum_{l=-\infty}^{\infty} \cos l y S^{-l} \boldsymbol{K}_{1}^{2} \boldsymbol{\Phi}^{(k)} .
\end{aligned}
$$

Therefore it holds that

$$
\iint_{D} J\left(\Delta z^{(0)}, \varphi^{(k)}\right) \Delta^{2} \Phi^{(k)} d x d y={\frac{2 \pi^{2}}{\alpha}}^{t} J_{1} \boldsymbol{K}_{1}^{2} \boldsymbol{\Phi}^{(k)} .
$$

In the same way, we have that

$$
\begin{aligned}
J\left(\Delta \varphi^{(k)}, z^{(0)}\right) & =\left(-k \alpha^{t} \boldsymbol{\varphi}^{(k)} \boldsymbol{K}_{1} \boldsymbol{s}(k \alpha)\right)\left({ }^{t} \boldsymbol{w}^{(0)} \boldsymbol{N}^{-4}(-\boldsymbol{N}) \boldsymbol{s}(0)\right) \\
& =k \alpha^{t} \boldsymbol{w}^{(0)} \boldsymbol{N}^{-3} \boldsymbol{s}(0) \boldsymbol{s}^{\boldsymbol{s}}(k \alpha) \boldsymbol{K}_{1} \boldsymbol{\varphi}^{(k)} \\
& =k \alpha^{t} \boldsymbol{w}^{(0)} \boldsymbol{N}^{-3} \frac{1}{2}\left(\boldsymbol{C}(-k \alpha)-\boldsymbol{C}^{\prime}(k \alpha)\right) \boldsymbol{K}_{1} \boldsymbol{\varphi}^{(k)} \\
& =\frac{k \alpha}{2} \sum_{l=-\infty}^{\infty} \cos (k \alpha x+l y)^{t} \boldsymbol{w}^{(0)} \boldsymbol{N}^{-3}(I-R) S^{l} \boldsymbol{K}_{1} \boldsymbol{\varphi}^{(k)} \equiv{ }^{t} \boldsymbol{J}_{2} \boldsymbol{c}(k \alpha),
\end{aligned}
$$

where

We obtain also that

$$
\left(\boldsymbol{J}_{2}\right)_{n} \equiv \frac{k \alpha}{2} \boldsymbol{w}^{(0)} \boldsymbol{N}^{-3}(I-R) S^{n} \boldsymbol{K}_{1} \boldsymbol{\varphi}^{(k)} .
$$

$$
\begin{aligned}
J\left(\Delta \varphi^{(k)}, z^{(0)}\right) \Delta^{2} \Phi^{(k)} & ={ }^{t} \boldsymbol{J}_{2} \boldsymbol{c}(k \alpha)^{t} \boldsymbol{c}(k \alpha) \boldsymbol{K}_{1}^{2} \boldsymbol{\Phi}^{(k)}=\frac{1}{2}{ }^{t} \boldsymbol{J}_{2}\left(\boldsymbol{C}^{\prime}(2 k \alpha)+\boldsymbol{C}(0)\right) \boldsymbol{K}_{1}^{2} \boldsymbol{\Phi}^{(k)} \\
& =\frac{1}{2}{ }^{t} \boldsymbol{J}_{2} \sum_{l=-\infty}^{\infty} \cos (2 k \alpha x+l y) R S^{l} \boldsymbol{K}_{1}^{2} \boldsymbol{\Phi}^{(k)}+\frac{1}{2} \boldsymbol{J}_{2} \sum_{l=-\infty}^{\infty} \cos l y S^{l} \boldsymbol{K}_{1}^{2} \boldsymbol{\Phi}^{(k)}
\end{aligned}
$$


Hence it holds that

$$
\iint_{D} J\left(\Delta \varphi^{(k)}, z^{(0)}\right) \Delta^{2} \Phi^{(k)} d x d y=\frac{2 \pi^{2}}{\alpha}{ }^{t} J_{2} \boldsymbol{K}_{1}^{2} \boldsymbol{\Phi}^{(k)},
$$

Proposition $2,\left(14^{\prime}\right),(15)$ and $\left(15^{\prime}\right)$ then yield

$$
\begin{aligned}
D_{1}^{(k)}= & \frac{2 \pi^{2}}{\alpha}\left({ }^{t} \boldsymbol{J}_{1}+{ }^{t} \boldsymbol{J}_{2}\right) \boldsymbol{K}_{1}^{2} \boldsymbol{\Phi}^{(k)} \\
= & k \pi^{2} \sum_{m=-\infty}^{\infty}{ }^{t} \boldsymbol{w}^{(0)}\left\{\boldsymbol{N}^{-1}(I-R) S^{m} \boldsymbol{\varphi}^{(k)}+\boldsymbol{N}^{-3}(I-R) S^{m} \boldsymbol{K}_{1} \boldsymbol{\varphi}^{(k)}\right\}(-1)^{m} b_{m} \\
= & \frac{k^{2} \pi^{2} \alpha \lambda_{k}}{2} \sum_{m=-\infty}^{\infty} \sum_{n=-\infty}^{\infty}{ }^{t} \boldsymbol{\varphi}^{(k)} \boldsymbol{K}_{1} n S^{n} \boldsymbol{\varphi}^{(k)}\left(\boldsymbol{N}^{-1}(I-R) S^{m} \boldsymbol{\varphi}^{(k)}\right. \\
& \left.+\boldsymbol{N}^{-3}(I-R) S^{m} \boldsymbol{K}_{1} \boldsymbol{\varphi}^{(k)}\right)_{n} \tilde{b}_{m},
\end{aligned}
$$

where $(M)_{n}$ stands for the $n$-th row vector of the matrix $M$. Hence we can rewrite

$$
D_{1}^{(k)}=\frac{k^{2} \pi^{2} \alpha \lambda_{k}}{2} \sum_{m=-\infty}^{\infty} \sum_{n=-\infty}^{\infty} w_{n} A_{n m} \tilde{b}_{m},
$$

where

$$
\begin{aligned}
w_{n} & \equiv{ }^{t} \boldsymbol{\varphi}^{(k)} \boldsymbol{K}_{1} n S^{n} \boldsymbol{\varphi}^{(k)}=n \sum_{j=-\infty}^{\infty}\left(-k_{j}\right) \varphi_{j} \varphi_{n+j}, \\
\tilde{b}_{m} & \equiv(-1)^{m} b_{m}=(-1)^{m}\left(k^{2} \alpha^{2}+m^{2}-1\right) \varphi_{m}, \quad \tilde{b}_{m}>0 \text { if } m \geq 0, \\
A_{n, m} & \equiv\left(N^{-1}(I-R) S^{m} \boldsymbol{\varphi}^{(k)}\right)_{n}+\left(N^{-3}(I-R) S^{m} \boldsymbol{K}_{1} \boldsymbol{\varphi}^{(k)}\right)_{n} .
\end{aligned}
$$

Here we have denoted $\varphi_{j}^{(k)}$ in $\left(4^{\prime \prime \prime}\right)$ simply by $\varphi_{j}$. Note that $k_{j}, \boldsymbol{\varphi}^{(k)}, \boldsymbol{K}_{1}, N^{-1}$ and $R$ are given in Proposition 4. We can easily see $w_{0}=0$. We also remark that $A_{n, m}=A_{-n, m}$. In fact, since

$$
\begin{aligned}
A_{n, m} & =\frac{1}{n}\left(-\varphi_{-n+m}+\varphi_{n+m}\right)+\frac{1}{n^{3}}\left(k_{-n+m} \varphi_{-n+m}-k_{n+m} \varphi_{n+m}\right) \\
& =\frac{1}{n^{3}}\left\{\left(-n^{2}+k_{-n+m}\right) \varphi_{-n+m}+\left(n^{2}-k_{n+m}\right) \varphi_{n+m}\right\},
\end{aligned}
$$

we have that

$$
\begin{aligned}
A_{-n, m} & =\frac{-1}{n^{3}}\left\{\left(-n^{2}+k_{n+m}\right) \varphi_{n+m}+\left(n^{2}-k_{-n+m}\right) \varphi_{-n+m}\right\} \\
& =\frac{1}{n^{3}}\left\{\left(-n^{2}+k_{-n+m}\right) \varphi_{-n+m}+\left(n^{2}-k_{n+m}\right) \varphi_{n+m}\right\}=A_{n, m} .
\end{aligned}
$$


Note that $A_{00}=0$. Hereafter we put $n>0$. Since $\varphi_{-m}=(-1)^{m} \varphi_{m}, \tilde{b}_{-m}=(-1)^{m} \tilde{b}_{m}$ and $k_{-m}=k_{m}$, we also have the following for $m \neq 0$ :

$$
\begin{aligned}
A_{n, m} \tilde{b}_{m}+A_{n,-m} \tilde{b}_{-m} & \\
= & \frac{1}{n^{3}} \tilde{b}_{m}\left\{\left(-n^{2}+k_{-n+m}\right) \varphi_{-n+m}+\left(n^{2}-k_{n+m}\right) \varphi_{n+m}\right\}\left\{1-(-1)^{n}\right\} \\
& = \begin{cases}0 & \text { if } n \text { is even, } \\
\frac{2}{n^{3}} \tilde{b}_{m}\left\{\left(-n^{2}+k_{-n+m}\right) \varphi_{-n+m}+\left(n^{2}-k_{n+m}\right) \varphi_{n+m}\right\} & \text { if } n \text { is odd. }\end{cases}
\end{aligned}
$$

Also, for $m=0$, it holds that

$$
\begin{aligned}
A_{n, 0} \tilde{b}_{0} & =\frac{1}{n^{3}}\left\{\left(-n^{2}+k_{-n}\right) \varphi_{-n}+\left(n^{2}-k_{n}\right) \varphi_{n}\right\} \\
& =\frac{1}{n^{3}}\left\{\left(-n^{2}+k_{n}\right)(-1)^{n} \varphi_{n}+\left(n^{2}-k_{n}\right) \varphi_{n}\right\}=\frac{1}{n^{3}}\left(n^{2}-k_{n}\right) \varphi_{n}\left\{1-(-1)^{n}\right\} \\
& = \begin{cases}0 & \text { if } n \text { is even, } \\
\frac{2}{n^{3}}\left(n^{2}-k_{n}\right) \varphi_{n} & \text { if } n \text { is odd. }\end{cases}
\end{aligned}
$$

Therefore we can rewrite $D_{1}^{(k)}$ as

$$
D_{1}^{(k)}=\frac{k^{2} \pi^{2} \alpha \lambda_{k}}{2} \sum_{n=1,3,5, \ldots}\left(w_{n}+w_{-n}\right)\left\{A_{n, 0} \tilde{b}_{0}+\sum_{m=1}^{\infty}\left(A_{n, m} \tilde{b}_{m}+A_{n,-m} \tilde{b}_{-m}\right)\right\} .
$$

Now, let us investigate the term for each odd $n \geq 1$. We first have

$$
A_{n, 0} \tilde{b}_{0}=\frac{2}{n^{3}}\left(n^{2}-k_{n}\right) \varphi_{n}=-\frac{2(k \alpha)^{2}}{n^{3}} \varphi_{n}>0 .
$$

Also, in the sum

$$
\sum_{m=1}^{\infty}\left(A_{n, m} \tilde{b}_{m}+A_{n,-m} \tilde{b}_{-m}\right)=\frac{2}{n^{3}} \sum_{m=1}^{\infty} \tilde{b}_{m}\left\{\left(-n^{2}+k_{-n+m}\right) \varphi_{-n+m}+\left(n^{2}-k_{n+m}\right) \varphi_{n+m}\right\},
$$

the term containing $\varphi_{0}$ is given by

$$
\frac{2 \tilde{b}_{n}\left(-n^{2}+k_{0}\right) \varphi_{0}}{n^{3}}>0 .
$$

If $\beta=k \alpha$ tends to 1 , only this term diverges to $+\infty$, since $\varphi_{0}=1 /\left(\beta^{2}-1\right)<0$ diverges and the sum of other terms is uniformly bounded. Hence we have $\sum_{m=1}^{\infty}\left(A_{n, m} \tilde{b}_{m}+A_{n,-m} \tilde{b}_{-m}\right)>$ 0 if $\beta$ is close to 1 .

In the case that $\beta \rightarrow 0, \varphi_{m}=\left(\beta^{2}+m^{2}-1\right)^{-1} b_{m}$ are very small if $m \neq 0$, since

$$
\left|\rho_{m}\right|<\frac{1}{a_{m}}=\frac{\lambda_{k} \beta\left(\beta^{2}+m^{2}-1\right)}{2\left(\beta^{2}+m^{2}\right)^{2}} \rightarrow 0 \quad \text { as } \beta \rightarrow 0,
$$


but $\left|\varphi_{0}\right|=\left|\beta^{2}-1\right|$ tends to 1 . So in this case the term with $\varphi_{0}$ is dominant, and we have

$$
\sum_{m=1}^{\infty}\left(A_{n, m} \tilde{b}_{m}+A_{n,-m} \tilde{b}_{-m}\right)>0 .
$$

On the other hand, it follows from ( $\left.4^{\prime \prime \prime}\right)$ that for each odd number $n \geq 1$

$$
\begin{aligned}
w_{n}+w_{-n} & =n \sum_{j=-\infty}^{\infty}\left(-k_{j}\right) \varphi_{j} \varphi_{n+j}+(-n) \sum_{j=-\infty}^{\infty}\left(-k_{j}\right) \varphi_{j} \varphi_{-n+j} \\
& =n \sum_{j=-\infty}^{\infty}\left(-k_{j}\right) \varphi_{j} \varphi_{n+j}+n \sum_{j=-\infty}^{\infty} k_{j+n} \varphi_{j+n} \varphi_{j} \\
& =n \sum_{j=-\infty}^{\infty}\left(k_{j+n}-k_{j}\right) \varphi_{j} \varphi_{n+j} \\
& =n\left\{2\left(k_{n}-k_{0}\right) \varphi_{0} \varphi_{n}+\sum_{j \neq 0,-n}\left(k_{j+n}-k_{j}\right) \varphi_{j} \varphi_{n+j}\right\} .
\end{aligned}
$$

If $\beta$ tends to 1 , only the first term $2 n^{3} \varphi_{0} \varphi_{n}$ diverges to $+\infty$ and the other terms are bounded. Then $w_{n}+w_{-n}>0$ holds as $\beta \rightarrow 1$. Note that, in the case where $\beta$ is close to $0, w_{n}+$ $w_{-n}>0$ follows directly, since $\varphi_{j}(j \neq 0)$ are very small. Consequently, summing up above estimates, we have $D_{1}^{(k)}>0$ when $\beta$ is close to 1 or 0 .

4.2. $D_{2}^{(k)}$ and its estimates. Next we investigate $D_{2}^{(k)}$ by using the Fourier expansion of each component function. First, $J\left(\Delta z^{(2 k)}, \varphi^{(k)}\right)$ turns out to be

$$
\begin{gathered}
\left.\left(-2 k \alpha^{t} z^{(2 k)} \boldsymbol{K}_{2} \boldsymbol{s}(2 k \alpha)\right){ }^{t} \boldsymbol{\varphi}^{(k)}(-\boldsymbol{N}) \boldsymbol{s}(k \alpha)\right)-\left({ }^{t} \boldsymbol{z}^{(2 k)} \boldsymbol{K}_{2}(-\boldsymbol{N}) \boldsymbol{s}(2 k \alpha)\right)\left(-k \alpha^{t} \boldsymbol{\varphi}^{(k)} \boldsymbol{s}(k \alpha)\right) \\
\quad=2 k \alpha^{t} \boldsymbol{z}^{(2 k)} \boldsymbol{K}_{2} \boldsymbol{s}(2 k \alpha)^{t} \boldsymbol{s}(k \alpha) \boldsymbol{N} \boldsymbol{\varphi}^{(k)}-k \alpha^{t} z^{(2 k)} \boldsymbol{K}_{2} \boldsymbol{N} \boldsymbol{s}(2 k \alpha)^{t} \boldsymbol{s}(k \alpha) \boldsymbol{\varphi}^{(k)} \\
=k \alpha^{t} \boldsymbol{z}^{(2 k)} \boldsymbol{K}_{2}\left(2 \boldsymbol{s}(2 k \alpha)^{t} \boldsymbol{s}(k \alpha) \boldsymbol{N}-\boldsymbol{N} \boldsymbol{s}(2 k \alpha)^{t} \boldsymbol{s}(k \alpha)\right) \boldsymbol{\varphi}^{(k)}
\end{gathered}
$$

where $\boldsymbol{K}_{2}$ is a diagonal matrix with $n$-th element $\left(\boldsymbol{K}_{2}\right)_{n} \equiv-\left(4 k^{2} \alpha^{2}+n^{2}\right)=-\tilde{k}_{n}$. In a manner similar to $J\left(\Delta \varphi^{(k)}, z^{(0)}\right), J\left(\Delta \varphi^{(k)}, z^{(2 k)}\right)$ becomes

$$
\begin{gathered}
\left(-k \alpha^{t} \boldsymbol{\varphi}^{(k)} \boldsymbol{K}_{1} \boldsymbol{s}(k \alpha)\right)\left({ }^{t} \boldsymbol{z}^{(2 k)}(-\boldsymbol{N}) \boldsymbol{s}(2 k \alpha)\right)-\left({ }^{t} \boldsymbol{\varphi}^{(k)} \boldsymbol{K}_{1}(-\boldsymbol{N}) \boldsymbol{s}(k \alpha)\right)\left(-2 k \alpha^{t} \boldsymbol{z}^{(2 k)} \boldsymbol{s}(2 k \alpha)\right) \\
\left.=k \alpha^{t} \boldsymbol{z}^{(2 k)}\left(\boldsymbol{N} \boldsymbol{s}(2 k \alpha){ }^{t} \boldsymbol{s}(k \alpha)-2 \boldsymbol{s}(2 k \alpha)\right)^{t} \boldsymbol{s}(k \alpha) \boldsymbol{N}\right) \boldsymbol{K}_{1} \boldsymbol{\varphi}^{(k)},
\end{gathered}
$$

where $\boldsymbol{K}_{1}$ is a diagonal matrix with $n$-th element $-\left(k^{2} \alpha^{2}+n^{2}\right)=-k_{n}$. Substituting

$$
\begin{aligned}
s(2 k \alpha)^{t} \boldsymbol{s}(k \alpha) & \equiv \frac{1}{2}(\boldsymbol{C}(k \alpha)-\boldsymbol{C}(3 k \alpha) R) \\
& =\frac{1}{2}\left\{\sum_{l=-\infty}^{\infty} \cos (k \alpha x+l y) S^{-l}-\sum_{l=-\infty}^{\infty} \cos (3 k \alpha x+l y) R S^{l}\right\},
\end{aligned}
$$

we obtain

$$
J\left(\Delta z^{(2 k)}, \varphi^{(k)}\right)={ }^{t} \boldsymbol{J}_{3} \boldsymbol{c}(k \alpha)+{ }^{t} \boldsymbol{J}^{\prime}{ }_{3} \boldsymbol{c}(3 k \alpha),
$$


where

$$
\begin{aligned}
\left(\boldsymbol{J}_{3}\right)_{n} & \equiv \frac{1}{2} k \alpha^{t} z^{(2 k)} \boldsymbol{K}_{2}\left(2 S^{-n} \boldsymbol{N}-\boldsymbol{N} S^{-n}\right) \boldsymbol{\varphi}(k) \\
\left(\boldsymbol{J}^{\prime}{ }_{3}\right)_{n} & \equiv \frac{1}{2} k \alpha^{t} \boldsymbol{z}^{(2 k)} \boldsymbol{K}_{2}\left(-2 R S^{n} \boldsymbol{N}+\boldsymbol{N} R S^{n}\right) \boldsymbol{\varphi}^{(k)} .
\end{aligned}
$$

Similarly, we also have

$$
\boldsymbol{J}\left(\Delta \varphi^{(k)}, z^{(2 k)}\right)={ }^{t} \boldsymbol{J}_{4} \boldsymbol{c}(k \alpha)+{ }^{t} \boldsymbol{J}^{\prime}{ }_{4} \boldsymbol{c}(3 k \alpha),
$$

where

$$
\begin{aligned}
\left(\boldsymbol{J}_{4}\right)_{n} & \equiv \frac{1}{2} k \alpha^{t} z^{(2 k)}\left(\boldsymbol{N} S^{-n}-2 S^{-n} \boldsymbol{N}\right) \boldsymbol{K}_{1} \boldsymbol{\varphi}^{(k)} \\
\left(\boldsymbol{J}^{\prime}{ }_{4}\right)_{n} & \equiv \frac{1}{2} k \alpha^{t} z^{(2 k)}\left(-\boldsymbol{N} R S^{n}+2 R S^{n} \boldsymbol{N}\right) \boldsymbol{K}_{1} \boldsymbol{\varphi}^{(k)}
\end{aligned}
$$

Thus we have

$$
\begin{aligned}
& \iint_{D} J\left(\Delta z^{(2 k)}, \varphi^{(k)}\right) \Delta^{2} \Phi^{(k)} d x d y=\frac{2 \pi^{2}}{\alpha}{ }^{t} J_{3} \boldsymbol{K}_{1}^{2} \boldsymbol{\Phi}^{(k)}, \\
& \iint_{D} J\left(\Delta \varphi^{(k)}, z^{(2 k)}\right) \Delta^{2} \Phi^{(k)} d x d y=\frac{2 \pi^{2}}{\alpha}{ }^{t} J_{4} \boldsymbol{K}_{1}^{2} \boldsymbol{\Phi}^{(k)} .
\end{aligned}
$$

Moreover, rewriting $\left(\boldsymbol{J}_{3}\right)_{n}$ and $\left(\boldsymbol{J}_{4}\right)_{n}$ by using Proposition 5 , we have

$$
\begin{aligned}
& \left(\boldsymbol{J}_{3}\right)_{n}=\frac{1}{2} k \alpha^{t} z^{(2 k)} \boldsymbol{K}_{2} S^{-n}(\boldsymbol{N}-n I) \boldsymbol{\varphi}^{(k)}, \\
& \left(\boldsymbol{J}_{4}\right)_{n}=\frac{1}{2} k \alpha^{t} z^{(2 k)} S^{-n}(-\boldsymbol{N}+n I) \boldsymbol{K}_{1} \boldsymbol{\varphi}^{(k)} .
\end{aligned}
$$

From Proposition 2, $\left(12^{\prime \prime}\right),\left(14^{\prime}\right),(17)$ and (18), it then follows that

$$
\begin{aligned}
D_{2}^{(k)}= & \frac{2 \pi^{2}}{\alpha}\left({ }^{t} \boldsymbol{J}_{3}+{ }^{t} \boldsymbol{J}_{4}\right) \boldsymbol{K}_{1}^{2} \boldsymbol{\Phi}^{(k)} \\
= & \frac{2 \pi^{2}}{\alpha} \sum_{m=-\infty}^{\infty}\left\{\frac{k}{2} \alpha^{t} \boldsymbol{z}^{(2 k)} \boldsymbol{K}_{2} S^{-m}(\boldsymbol{N}-m I) \boldsymbol{\varphi}^{(k)}+\frac{k}{2} \alpha^{t} z^{(2 k)} S^{-m}(-\boldsymbol{N}+m I) \boldsymbol{K}_{1} \boldsymbol{\varphi}^{(k)}\right\} \tilde{b}_{m} \\
= & k \pi^{2} \sum_{m=-\infty}^{\infty}{ }^{t} z^{(2 k)}\left(\boldsymbol{K}_{2} S^{-m}-S^{-m} \boldsymbol{K}_{1}\right)(\boldsymbol{N}-m I) \boldsymbol{\varphi}^{(k)} \tilde{b}_{m} \\
= & \frac{k^{2} \pi^{2} \alpha \lambda_{k}}{2} \sum_{m, n=-\infty}^{\infty}{ }^{t} \boldsymbol{\varphi}^{(k)} \boldsymbol{K}_{1}(2 \boldsymbol{N}-n I) R S^{n} \boldsymbol{\varphi}^{(k)} \\
& \times\left(\boldsymbol{D}^{-1} \boldsymbol{\Lambda}^{-1}\left(\boldsymbol{K}_{2} S^{-m}-S^{-m} \boldsymbol{K}_{1}\right)(\boldsymbol{N}-m I) \boldsymbol{\varphi}^{(k)}\right)_{n} \tilde{b}_{m} .
\end{aligned}
$$

Hence we obtain

$$
D_{2}^{(k)}=\frac{k^{2} \pi^{2} \alpha \lambda_{k}}{2} \sum_{m=-\infty}^{\infty} \sum_{n=-\infty}^{\infty} \tilde{w}_{n} \tilde{A}_{n m} \tilde{b}_{m},
$$


where we put

$$
\begin{aligned}
\tilde{w}_{n} & \equiv{ }^{t} \boldsymbol{\varphi}^{(k)} \boldsymbol{K}_{1}(2 \boldsymbol{N}-n I) R S^{n} \boldsymbol{\varphi}^{(k)}=\sum_{j=-\infty}^{\infty} k_{j}(n-2 j) \varphi_{j} \varphi_{n-j}, \\
\tilde{A}_{n m} & \equiv\left(\boldsymbol{D}^{-1} \boldsymbol{\Lambda}^{-1}\left(\boldsymbol{K}_{2} S^{-m}-S^{-m} \boldsymbol{K}_{1}\right)(\boldsymbol{N}-m I) \boldsymbol{\varphi}^{(k)}\right)_{n} \\
& =\sum_{j=-\infty}^{\infty} d_{n}^{(j)} \ddot{a}_{j}^{-1}\left(-\tilde{k}_{j}+k_{j-m}\right)(-2 m+j) \varphi_{j-m}
\end{aligned}
$$

Note that $d_{n}^{(j)}$ is given in Proposition 6 and $\ddot{a}_{j}^{-1}$ is equal to $\lambda_{k} k \alpha\left(4 k^{2} \alpha^{2}+n^{2}-1\right)$. In $D_{2}^{(k)}$, the term containing $\varphi_{0}^{2}$ is given by

$$
\tilde{D}_{2}^{(k)}=\frac{k^{2} \pi^{2} \alpha \lambda_{k}}{2} \sum_{m=-\infty}^{\infty} \sum_{n=-\infty}^{\infty}-n^{3} \varphi_{n} m d_{n}^{(m)} \ddot{a}_{m}^{-1}\left(3 \beta^{2}+m^{2}\right) \tilde{b}_{m} \varphi_{0}^{2},
$$

while we already know the corresponding term in $D_{1}^{(k)}$ is given by

$$
\tilde{D}_{1}^{(k)}=\frac{k^{2} \pi^{2} \alpha \lambda_{k}}{2} \sum_{n=1,3,5, \ldots} 4\left(n^{2}-\beta^{2}\right) \varphi_{n} b_{n} \varphi_{0}^{2}>0 .
$$

First, we compare these values as $\beta$ tends to 1 . Hereafter we neglect $\left(k^{2} \pi^{2} \alpha \lambda_{k} / 2\right) \varphi_{0}^{2}$, which is a positive factor involved in both $\left(19^{\prime}\right)$ and $\left(16^{\prime}\right)$. Now, recall the relation $\varphi_{n}=$ $\left(\beta^{2}+n^{2}-1\right)^{-1} b_{n}$ and note the following limits as $\beta \rightarrow 1$ :

$$
\frac{n^{3}}{\beta^{2}+n^{2}-1} \rightarrow n, \quad \frac{3 \beta^{2}+m^{2}}{\beta\left(4 \beta^{2}+m^{2}-1\right)} \rightarrow 1
$$

Then we can regard that $\tilde{D}_{2}^{(k)} /\left\{\left(k^{2} \pi^{2} \alpha \lambda_{k} / 2\right) \varphi_{0}^{2}\right\}$ is close to

$$
\sum_{m=-\infty}^{\infty} \sum_{n=-\infty}^{\infty}(-1)^{m+1} n m \frac{d_{n}^{(m)}}{\lambda} b_{n} b_{m} \equiv \sum_{m=-\infty}^{\infty} \sum_{n=-\infty}^{\infty} \tilde{c}_{m, n}
$$

where

$$
\tilde{c}_{m, n} \equiv(-1)^{m+1} n m \frac{d_{n}^{(m)}}{\lambda} b_{n} b_{m}
$$

with $\lambda=\lambda_{k}=\lambda(k \alpha)=\lambda(\beta), d_{n}^{(m)}=d_{n}^{(m)}(\beta)$ and $b_{n}=b_{n}(\beta)$. Note also that $\beta$ is a monotone increasing function of $\lambda$, and $\tilde{c}_{m, n}$ has the symmetric properties such as

$$
\tilde{c}_{m, n}=\tilde{c}_{-m,-n}, \quad \tilde{c}_{m, n}=\tilde{c}_{n, m} .
$$


In fact, we have

$$
\begin{aligned}
\tilde{c}_{-m,-n} & =(-1)^{-m+1}(-n)(-m) \frac{d_{-n}^{(-m)}}{\lambda} b_{-n} b_{-m} \\
& =(-1)^{-m+1} n m(-1)^{n+m} \frac{d_{n}^{(m)}}{\lambda}(-1)^{n} b_{n}(-1)^{m} b_{m}=\tilde{c}_{m, n}, \\
\tilde{c}_{n, m} & =(-1)^{n+1} m n \frac{d_{m}^{(n)}}{\lambda} b_{m} b_{n}=(-1)^{n+1} m n(-1)^{n+m} \frac{d_{n}^{(m)}}{\lambda} b_{n} b_{m}=\tilde{c}_{m, n} .
\end{aligned}
$$

Here we have used $d_{-n}^{(-m)}=(-1)^{n+m} d_{n}^{(m)}$ and $d_{m}^{(n)}=(-1)^{n+m} d_{n}^{(m)}$.

Therefore it suffices to deal with the terms with $m \geq|n|$. Then (21) becomes

$$
\begin{aligned}
\sum_{m=-\infty}^{\infty} \sum_{n=-\infty}^{\infty} \tilde{c}_{m, n}= & 2 \sum_{m=1}^{\infty} \tilde{c}_{m, m}+4 \sum_{n=1}^{\infty} \sum_{m=n+1}^{\infty} \tilde{c}_{m, n} \\
& +2 \sum_{m=1}^{\infty} \tilde{c}_{m,-m}+4 \sum_{n=1}^{\infty} \sum_{m=n+1}^{\infty} \tilde{c}_{m,-n}
\end{aligned}
$$

since this series is absolutely convergent. Now note that the sign of $\tilde{c}_{m, n}$ is alternating with respect to $m$, since

$$
\tilde{c}_{m, n}=(-1)^{m+1} m n \frac{1}{\lambda} \prod_{i=n+1}^{m} \eta_{i}^{+} N_{n+1}^{-1}\left|b_{m}\right|\left|b_{n}\right| .
$$

For our convenience of calculation, we rewrite (24) as follows:

(26) $\bar{D}_{2}^{(k)} \equiv \sum_{m, n=-\infty}^{\infty} \tilde{c}_{m, n}=2 \sum_{n=1}^{\infty}\left\{\sum_{m=n}^{\infty} \tilde{c}_{m, n}+\sum_{m=n+1}^{\infty} \tilde{c}_{m, n}+\sum_{m=n}^{\infty} \tilde{c}_{m,-n}+\sum_{m=n+1}^{\infty} \tilde{c}_{m,-n}\right\}$.

Our aim now is to show that the absolute value of (26) is smaller than the following sum

$$
\bar{D}_{1}^{(k)} \equiv \sum_{n=1,3,5, \ldots} \frac{n^{2}-1}{n^{2}} b_{n}^{2}=\sum_{n=3,5, \ldots} \frac{n^{2}-1}{n^{2}} b_{n}^{2},
$$

which corresponds to $\tilde{D}_{1}^{(k)} /\left\{\left(k^{2} \pi^{2} \alpha \lambda_{k} / 2\right) \varphi_{0}^{2}\right\}$ as $\beta$ tends to 1 . To this end, we investigate continued fractions and related sequences in detail in the next section.

\section{Quasi-monotonicity and supercriticality.}

5.1. Sequences generated by continued fractions. In this section we consider, in a general situation, properties of continued fractions related to a sequence $\{a(n)\}_{1}^{\infty}$ of positive numbers, which is supposed to have a parameter $\lambda \in\left(\lambda_{0}, \infty\right), \lambda_{0}>0$. More precisely, we put $a(n)=a(n, \lambda)$. The continued fraction $\rho(n)=\rho(n, \lambda)$ defined by

$$
\rho(n, \lambda)=\frac{1}{a(n)+\rho(n+1, \lambda)}=\frac{1}{a(n)}+\frac{1}{a(n+1)}+\cdots
$$

becomes a positive valued sequence satisfying $\rho(n, \lambda)<1 / a(n, \lambda)$. We are interested in the behavior of $\rho(n, \lambda)$ as $\lambda$ tends to infinity. As seen before, $\{\rho(n, \lambda)\}_{1}^{\infty}$ gives rise to a new 
sequence $\{b(n)\}_{1}^{\infty}=\{b(n, \lambda)\}_{1}^{\infty}$ defined by $b(n, \lambda) \equiv \prod_{i=1}^{n} \rho(i, \lambda)$ for $n \geq 1$. Note that $b(n+1)=b(n) \rho(n+1)$ for $n \in \mathbf{N}$ and $b(0)=1$. On the other hand, we can see that $\{\rho(n)\}$ satisfies, for all $n \in \mathbf{N}$,

$$
\left(1+a(n) a(n+1)+\left(a_{n} / a_{n+2}\right)\right)^{-1}<\rho(n) \rho(n+1)<(1+a(n) a(n+1))^{-1}<1 .
$$

The above inequality shows that the sequence of even terms and that of odd terms are strictly monotone decreasing, respectively. In the previous sections, we considered the above sequences with $a(n), \rho(n)$ and $b(n)$ replaced respectively by $a_{n}=a_{n}(\beta(\lambda), \lambda),\left|\rho_{n}\right|$ and $(-1)^{n} b_{n}$. We note that the inverse function $\beta=\beta(\lambda)$ of $\lambda(\beta)$ is a monotone increasing continuous function defined on $(\sqrt{2}, \infty)$. Therefore we can regard $a_{n}(\beta(\lambda), \lambda)=a(n, \lambda)$ as a sequence having one parameter $\lambda$.

In our analysis, which is closely related to Abel's criterion for summability, we are concerned whether $\{b(n)\}=\{b(n, \lambda)\}$ is a monotone decreasing sequence or not, that is, if $\rho(n)<1$ holds for all $n \in \mathbf{N}$. Although this is not true in general, $b(n)=b(n, \lambda)$ has a property which is close to monotonicity for sufficiently large $\lambda$, which we call later quasimonotonicity.

In order to prove this property, we generalize $\rho(n, \lambda)$, which is defined first on $\mathbf{N} \times$ $\left(\lambda_{0}, \infty\right)$, to a continuous function $\rho(t, \lambda)$ defined on $[1, \infty) \times\left(\lambda_{0}, \infty\right)$, and use infinitesimal calculus. For this purpose, we assume that the extended function $a(t, \lambda)$ is a smooth function defined on $[1, \infty) \times\left(\lambda_{0}, \infty\right)$ satisfying

$$
\left\{\begin{array}{l}
\frac{t^{2}}{M^{2}} \leq a(t, \lambda) \leq \frac{c t^{2}}{M^{2}}, \quad \frac{d}{d t} a(t, \lambda) \leq \frac{c t}{M^{2}} \\
0 \leq \frac{d^{2}}{d t^{2}} a(t, \lambda), \quad 1 \leq t, \quad t \in[1, \infty), \quad \lambda \in\left(\lambda_{0}, \infty\right)
\end{array}\right.
$$

for some positive constant $c$, where $M=[\sqrt{\lambda}]+1$. Now, we remark two examples:

$$
\left\{\begin{array}{l}
a^{(+1)}(t, \lambda)=2\left(\beta(\lambda)^{2}+(t+1)^{2}\right)^{2} /\left(\lambda \beta(\lambda)\left(\beta(\lambda)^{2}+(t+1)^{2}-1\right)\right), \\
\tilde{a}(t, \lambda)=\left(4 \beta(\lambda)^{2}+t^{2}\right)^{2} /\left(\lambda \beta(\lambda)\left(4 \beta(\lambda)^{2}+t^{2}-1\right)\right) .
\end{array}\right.
$$

In particular, for all $n \in \mathbf{N}$, we have $a^{(+1)}(n, \lambda)=a_{n+1}(\beta(\lambda), \lambda), \tilde{a}(n, \lambda)=\tilde{a}_{n}(\beta(\lambda), \lambda)=$ $a_{n}(2 \beta(\lambda), \lambda)$. Note that $a^{(+1)}(t, \lambda)$ corresponds to $\left\{a_{n}(\beta(\lambda), \lambda)\right\}_{n=2}^{\infty}$, where we can take $c=$ 2 for large $\lambda_{0}$. Remark that, for $\left\{a_{n}(\beta(\lambda), \lambda)\right\}_{n=1}^{\infty}$, the extended function $a(t, \lambda)$ does not satisfies the third condition in (28). Therefore the term concerned with $n=1$ must be treated separately.

Now, we can define the extension $\rho(t)=\rho(t, \lambda)$ of $\rho(n, \lambda)$ by

$$
\rho(t) \equiv \frac{1}{a(t)}+\frac{1}{a(t+1)}+\cdots .
$$

Then from $\rho(t)=(a(t)+\rho(t+1))^{-1}$ it follows that $\rho(t)<1$ for $t>M$ and $\rho(t) \rho(t+1) \leq$ $(1+a(t) a(t+1))^{-1}<1$ for any $(t, \lambda) \in[1, \infty) \times\left(\lambda_{0}, \infty\right)$.

First, let us prove that $\rho(t)$ is continuous. Namely, setting $\mu(t, h) \equiv \rho(t+h)-\rho(t)$, we prove that $\lim _{h \rightarrow 0} \mu(t, h)=0$. Using the formula $\rho(t)=1 /\{a(t)+\rho(t+1)\}$ and the 
notation $d(t, h) \equiv a(t+h)-a(t)$, we obtain

$$
\begin{aligned}
&|\mu(t, h)|=|-\{d(t, h)+\mu(t+1, h)\} \rho(t+h) \rho(t)| \\
&= \mid-d(t, h) \rho(t+h) \rho(t) \\
&+\{d(t+1, h)+\mu(t+2, h)\} \rho(t+h) \rho(t) \rho(t+h+1) \rho(t+1) \mid \\
& \leq\left|\sum_{j=0}^{2 N-1}(-1)^{j+1}\left\{d(t+j, h) \prod_{i=0}^{j} \rho(t+h+i) \rho(t+i)\right\}\right| \\
&+\left|\mu(t+2 N, h) \prod_{i=0}^{2 N-1} \rho(t+h+i) \rho(t+i)\right| .
\end{aligned}
$$

Here we note that, for $k \in \mathbf{N}$,

$$
v_{j}(t, h) \equiv \prod_{i=0}^{j} \rho(t+h+i) \rho(t+i) \leq\left\{\begin{array}{l}
\rho(t+h+j) \rho(t+j) \quad \text { if } j=2 k, \\
1 \quad \text { if } j=2 k-1 .
\end{array}\right.
$$

Then, since $\lim _{t \rightarrow \infty} \rho(t)=0$, the second term of (30) converges to zero as $N$ tends to $\infty$. In consequence, by a standard $\varepsilon-\delta$ method, $\lim _{h \rightarrow 0}|\mu(t, h)|=0$ follows from $\lim _{h \rightarrow 0} d(t+j, h)=0$ for finite $j$.

We now define the sequence $\{b(n)\}=\{b(n, \lambda)\}$ by (10) with $\rho_{n}=\rho(n, \lambda)$. Denoting $\{b(n)\}$ by $\left|b_{n}\right|$ as before, we see that it has exponential decay as follows:

$$
\left|b_{n}\right|<\left|b_{n^{\prime}}\right| \exp \left\{-M^{-4}\left(n-n^{\prime}\right)^{5} / 20\right\} \text { for even positive integers } n^{\prime}<n \leq M .
$$

Similarly, for any even positive integers $r^{\prime} M<r M \leq M$, we have

$$
\left|b_{r M}\right|<\left|b_{r^{\prime} M}\right| \exp \left\{-\frac{M}{2} \int_{r^{\prime}}^{r} \log \left(1+s^{4}\right) d s\right\}, \quad\left|b_{r M}\right|=b(r M, \lambda) .
$$

Let us prove (32). First, from (28) and (29), the following relation holds:

$$
\frac{\left|b_{n+2}\right|}{\left|b_{n}\right|}=\left|\rho_{n+1} \rho_{n+2}\right|<\frac{1}{1+a_{n+1} a_{n+2}}<\frac{1}{1+((n+1) / M)^{4}}, \quad n \geq 0 .
$$

If $n=r M$ is even, then

$$
\left|b_{r M}\right|<\left|b_{0}\right|\left\{1 /\left(1+(1 / M)^{4}\right)\right\}\left\{1 /\left(1+(3 / M)^{4}\right)\right\} \cdots\left\{1 /\left(1+\{(r M-1) / M\}^{4}\right)\right\} .
$$

Since $b_{0}=1$, we have

$$
\log \left|b_{r M}\right|<-\sum_{k=1}^{r M / 2} \log \left\{1+((2 k-1) / M)^{4}\right\}<-\frac{M}{2} \int_{0}^{r} \log \left(1+s^{4}\right) d s .
$$

Since we have $\left(\log \left(1+s^{4}\right)\right)^{\prime}>2 s^{3}$ and $\log \left(1+s^{4}\right)>s^{4} / 2$ for $0<s<1$, it follows that $\int_{0}^{r} \log \left(1+s^{4}\right) d s>r^{5} / 10$ for $0<r<1$. Therefore we have

$$
\left|b_{r M}\right|<\exp \left(-M r^{5} / 20\right) \text { for even } r M=n \leq M .
$$

Also, in a manner similar to (34), it holds that if $n-n^{\prime}=r M-r^{\prime} M$ is even,

$$
\left|b_{r M}\right|<\left|b_{r^{\prime} M}\right| \exp \left\{-M\left(r-r^{\prime}\right)^{5} / 20\right\} .
$$


We have (32) immediately from $\left(34^{\prime}\right)$. Therefore, for odd $n=r M$, we have likewise

$$
\log \left|b_{r M}\right|<\log \left|b_{1}\right|-\frac{M}{2} \int_{1 / M}^{r} \log \left(1+s^{4}\right) d s<\log \left|b_{1}\right|-\frac{M r^{5}}{20}+\frac{1}{20 M^{4}} .
$$

Hence, we have the estimate of exponential decay

$$
\left|b_{r M}\right|<\left|b_{1}\right| \exp \left(1 / 20 M^{4}\right) \exp \left(-M r^{5} / 20\right) \text { for odd } n=r M \text {. }
$$

Note that it follows from Lemma A-3 that $\left|b_{1}\right|=|\rho(1, \lambda)|<(1+2 c)^{1 / 2}$, since it holds that $a(n+1, \lambda) / a(n, \lambda)<1+2 c$ for all $t$ and $\lambda$. Now we state the following theorem.

THEOREM 5. Under the assumption (28), $\{b(n, \lambda)\}_{n=1}^{\infty}$ defined by (10) with $\rho_{n}=$ $\rho(n, \lambda)$ satisfies $b(t, \lambda)>b(t+2, \lambda)$ and has the exponential decay property:

$$
b(n, \lambda)<C_{1} \exp \left\{-\frac{\lambda^{-2} n^{5}}{20}\right\}, \quad n \geq 1
$$

for some $C_{1}>0$. Moreover, there exists a constant $C>0$, such that $\rho(t, \lambda)$ satisfies the estimate

$$
\limsup _{\lambda \rightarrow \infty} S(\lambda) \lambda^{1 / 3} \leq C,
$$

where $S(\lambda) \equiv \sup _{1 \leq t<\infty}(\rho(t, \lambda)-1)$. Incidentally it holds that

$$
\max \left\{\rho(t, \lambda) ; t \geq 1, \lambda \in\left(\lambda_{0}, \infty\right)\right\}<(1+2 c)^{1 / 2} .
$$

The proof for the exponential decay is similar to the proof above. In order to show (35), we first consider the open region $I=\{t \in[1, \infty) ; \rho(t)>1\}$, which is divided into the countable union of open intervals $I=\sum_{k=1} I_{k}$, each $I_{k}$ being a connected component of $I$. Note that the number of $I_{k}$ satisfying $\left|I_{k}\right|>1 / j>0$ does not exceed $j(M-1)$ for any integer $j \geq 1$, since $I$ is contained in $[1, M)$. The length $\left|I_{k}\right|$ of each $I_{k}$ satisfies $\left|I_{k}\right|<1$. Indeed, if $\left|I_{k}\right| \geq 1$, then we have a point $t_{1}$ satisfying $\rho\left(t_{1}\right) \rho\left(t_{1}+1\right) \geq 1$, which is a contradiction.

Let us first study the values of $\lambda$ such that $S(\lambda)>0$. Since $\rho(t, \lambda)$ is continuous and $\rho(t, \lambda)$ tends to zero as $t \rightarrow \infty$, we examine the $\rho(t, \lambda)-1$ in $\left(t_{0}, M\right)$, supposing that $\rho\left(t_{0}^{\prime}, \lambda\right)-1>0$ is the maximum. For simplicity, let us denote $t_{0}^{\prime}$ by $t_{0}$ and omit $\lambda$. There exists a positive number $h=h(\lambda)<1$ such that $\rho\left(t_{0}+h\right)=1$. We first consider the value $\left|\rho\left(t_{0}\right)-1\right|=\left|\mu\left(t_{0}, h\right)\right|=\left|\rho\left(t_{0}+h\right)-\rho\left(t_{0}\right)\right|$. Then (30) yields

$$
\left|\rho\left(t_{0}\right)-1\right|<\left|\sum_{j=0}^{N-1}(-1)^{j} d\left(t_{0}+j, h\right) v_{j}\left(t_{0}, h\right)\right|+\left|\mu\left(t_{0}+N, h\right)\right|\left|v_{N-1}\left(t_{0}, h\right)\right| .
$$

Now we define $N$ to be $N=\min \left\{2 k \in 2 \mathbf{N} ; 2 k>M^{1+\delta}\right\}$, where $\delta>0$ is determined later. Then, from $\left|v_{N-1}\left(t_{0}, h\right)\right|<1$ it follows that

$$
\left|\mu\left(t_{0}+N, h\right)\right|\left|v_{N-1}\left(t_{0}, h\right)\right| \leq \frac{1}{a\left(t_{0}+N\right)}<M^{2} / N^{2}<M^{-2 \delta} .
$$


In order to estimate the first term in (36), modifying $v_{j}=v_{j}\left(t_{0}, h\right)$, we define a monotone decreasing sequence $\left\{\tilde{v}_{j}\right\}$ :

$$
\begin{aligned}
& \tilde{v}_{0} \equiv v_{0}=\rho\left(t_{0}\right)>1, \quad \tilde{v}_{1} \equiv v_{1}=\rho\left(t_{0}\right) \rho\left(t_{0}+h\right) \rho\left(t_{0}+1\right) \rho\left(t_{0}+h+1\right)<1, \\
& \tilde{v}_{j} \equiv \begin{cases}v_{j-1} & \text { if } \rho\left(t_{0}+j\right) \rho\left(t_{0}+h+j\right)>1, \\
v_{j} & \text { otherwise. }\end{cases}
\end{aligned}
$$

Since $\rho\left(t_{0}+j\right)<1$ for $t_{0}+j \geq M$, we have $\tilde{v}_{j}=v_{j}$ in this case. Moreover, it follows from the definition of $S(\lambda)$ that $v_{j}-\tilde{v}_{j} \leq\left\{(1+S(\lambda))^{2}-1\right\} \tilde{v}_{j}$. Note also that $v_{j}>\tilde{v}_{j}$ implies $\tilde{v}_{j+1}=v_{j+1}$, since $\rho(t) \rho(t+1)<1$ holds. Hence we obtain

$$
\begin{aligned}
& \left|\sum_{j=0}^{N-1}(-1)^{j} d\left(t_{0}+j, h\right) v_{j}\right| \\
& \quad \leq\left|\sum_{j=0}^{N-1}(-1)^{j} d\left(t_{0}+j, h\right) \tilde{v}_{j}\right|+\left|\sum_{j=2}^{M-t_{0}}(-1)^{j} d\left(t_{0}+j, h\right)\left(\tilde{v}_{j}-v_{j}\right)\right| .
\end{aligned}
$$

Note that for $t>M, 1 \leq a(t), \rho(t)<1$ and $\tilde{v}_{j}-v_{j}=0$. For the first term of (37) we use Abel's criterion for summability:

$$
\left(\inf _{k} \sum_{j=1}^{k} a_{j}\right) b_{1} \leq \sum_{j=1}^{n} a_{j} b_{j} \leq\left(\sup _{k} \sum_{j=1}^{k} a_{j}\right) b_{1},
$$

where $\left\{b_{j}\right\}$ is a monotone decreasing positive sequence. If $\left\{b_{j}\right\}$ is increasing, we have (38) with $a_{j}$ and $b_{1}$ replaced by $a_{n-j+1}$ and $b_{n}$, respectively.

Now, by the mean value theorem, $d\left(t_{0}+j, h\right)=a^{\prime}\left(t_{0}+j+\theta h\right) h$ for some $\theta=\theta(j) \in$ $(0,1)$. Thus the monotonicity of $a^{\prime}(t)$ implies that $\left\{d\left(t_{0}+j, h\right)\right\}$ is a monotone increasing sequence, since $0<h<1$. Then the following estimate follows from (38)

$$
\left|\sum_{j=0}^{k}(-1)^{j} d\left(t_{0}+j, h\right)\right| \leq d\left(t_{0}+k, h\right), \quad k \leq N-1 .
$$

Applying again Abel's criterion to the first term of (37), since $t_{0} \leq M$, it follows from (28) and (39) that

$$
\begin{aligned}
& \left|\sum_{j=0}^{N-1}(-1)^{j} d\left(t_{0}+j, h\right) \tilde{v}_{j}\right| \leq\left|d\left(t_{0}+N-1, h\right)\right| v_{0}=\left|a^{\prime}\left(t_{0}+N-1+h \theta\right)\right| h \rho\left(t_{0}\right) \\
& \quad<\frac{c}{M^{2}}\left(t_{0}+N-1+h \theta\right) \rho\left(t_{0}\right)<c\left(M^{-1}+M^{-1+\delta}+M^{-2}\right)\left(S\left(\lambda, t_{0}\right)+1\right) .
\end{aligned}
$$

Here we used the increasing order of $a^{\prime}(t)$ assumed in (28) and put $S\left(\lambda, t_{0}\right) \equiv$ $\sup _{t_{0} \leq t<\infty}(\rho(t, \lambda)-1)$. Suppose that $t_{0}$ satisfies $2 c(1+p)\left(M-t_{0}\right) / M=1 / 3$, where $p=$ $(1+2 c)^{1 / 2}$. Then it follows from (28) and the inequality $v_{j}-\tilde{v}_{j} \leq\left\{\left(1+S\left(\lambda, t_{0}\right)\right)^{2}-1\right\} \tilde{v}_{j}<$ 
$3 S\left(\lambda, t_{0}\right) \tilde{v}_{j}<6 S\left(\lambda, t_{0}\right)$ that

$$
\begin{aligned}
& \left|\sum_{j=2}^{M-t_{0}}(-1)^{j} a^{\prime}\left(t_{0}+j+h \theta_{j}\right) h\left(\tilde{v}_{j}-v_{j}\right)\right| \\
& \quad<\left(M-t_{0}\right) \frac{2 c}{M}\left(\left\{\left(1+S\left(\lambda, t_{0}\right)\right)^{2}-1\right\}\right)<\frac{1}{3} S\left(\lambda, t_{0}\right) .
\end{aligned}
$$

Note that, since $S(\lambda)<p-1$, we have $v_{j}-\tilde{v}_{j}<\left(\left\{\left(1+S\left(\lambda, t_{0}\right)\right)^{2}-1\right\}\right) \tilde{v}_{j}<(1+p) S\left(\lambda, t_{0}\right)$.

Combining (36), (36'), (37), (40), (41) and the above inequality, we obtain

$$
S\left(\lambda, t_{0}\right)<M^{-2 \delta}+\frac{2 c}{M^{1-\delta}}\left(S\left(\lambda, t_{0}\right)+1\right)+\frac{1}{3} S\left(\lambda, t_{0}\right) .
$$

Here put $\delta=1 / 3$ and suppose that $2 c / M^{1-\delta}<1 / 3$ for large $\lambda$. Then there exists a positive constant $M_{0}$ such that for $M>M_{0}$

$$
S\left(\lambda, t_{0}\right)<3(1+2 c) M^{-2 / 3}, \quad M=M(\lambda)=\{[\sqrt{\lambda}]+1\}^{-2 / 3},
$$

from which we obtain, for $M>M_{0}$,

$$
S\left(\lambda, t_{1}\right)<M^{-2 \delta}+\frac{2 c}{M^{1-\delta}}\left(S\left(\lambda, t_{1}\right)+1\right)+S\left(\lambda, t_{1}\right) / 3+S\left(\lambda, t_{0}\right) / 3, \quad t_{1}=t_{0}-\left(M-t_{0}\right) .
$$

Therefore we have

$$
S\left(\lambda, t_{1}\right) \leq 3(1+2 c) M^{-2 / 3}(1+(1 / 3)), \quad \text { for } M>M_{0} .
$$

Repeating this process $\left[M /\left(M-t_{0}\right)\right]+1$ times, we obtain (35) with

$$
C=3(1+2 c)\left(1+\frac{1}{3}\right)^{k_{0}}, \quad k_{0}=\left[6 c\left\{1+(1+2 c)^{1 / 2}\right\}\right]+1 .
$$

5.2. Positiveness of $\mu_{k}^{\prime \prime}(0)$. Now we return to the proof of Theorem 2. From (25), $\tilde{c}_{m, n}$ change signs alternately in $m$. The series $\sum_{n=-\infty}^{\infty} \sum_{m=-\infty}^{\infty} \tilde{c}_{m, n}$ converge absolutely. Hence, by (26), we obtain

$$
\begin{aligned}
\left|\sum_{n=-\infty}^{\infty} \sum_{m=-\infty}^{\infty} \tilde{c}_{m, n}\right| \leq & 2 \sum_{n=1}^{\infty}\left|\sum_{m=n}^{\infty} \tilde{c}_{m, n}\right|+2 \sum_{n=1}^{\infty}\left|\sum_{m=n+1}^{\infty} \tilde{c}_{m, n}\right| \\
& +2 \sum_{n=1}^{\infty}\left|\sum_{m=n}^{\infty} \tilde{c}_{m,-n}\right|+2 \sum_{n=1}^{\infty}\left|\sum_{m=n+1}^{\infty} \tilde{c}_{m,-n}\right| .
\end{aligned}
$$

We decompose the first term in the right-hand side into

$$
\sum_{n=1}^{\infty}\left|\sum_{m=n}^{\infty} \tilde{c}_{m, n}\right| \leq\left|\tilde{c}_{1,1}\right|+\left|\sum_{m=2}^{\infty} \tilde{c}_{m, 1}\right|+\sum_{n=2}^{\infty}\left|\sum_{m=n}^{\infty} \tilde{c}_{m, n}\right| .
$$

First, let us consider

$$
\sum_{m=n}^{\infty} \tilde{c}_{m, n}=n \frac{1}{\lambda} N_{n+1}^{-1}\left|b_{n}\right| P_{n}, \quad P_{n}=\sum_{m=n}^{\infty}(-1)^{m+1} m \prod_{i=n+1}^{m} \eta_{i}^{+}\left|b_{m}\right|,
$$


where $\prod_{i=n+1}^{m} \eta_{i}^{+}=1$ for $m=n$. Note that for $k=1,2, \ldots, a_{k+1}=a_{k+1}(\lambda)=a^{(+1)}(k, \lambda)$ satisfies the conditions (28). Therefore we consider the case where $n \geq 2$. The term $\tilde{c}_{1,1}$ can be estimated separately at the final part of this section.

Now, we verify

$$
0<\max _{-\infty<n<\infty} N_{n+1}^{-1}<C,
$$

in the following way. First note that $N_{n+1} \equiv \eta_{n+1}^{+}-\eta_{n+1}^{-}>\max \left\{\eta_{n+1}^{+}, \tilde{a}_{n}\right\}$. Then, for $n \geq M$ it holds that $N_{n+1} \geq \tilde{a}_{n} \geq 1$. For $0 \leq n<M$ we have $\eta_{n+1}^{+}>1 / 6$, since $\eta_{n+2}^{+}<2$ and $\eta_{n+1}^{+} \eta_{n+2}^{+}>\left(1+\tilde{a}_{n+1} \tilde{a}_{n+2}+\left(\tilde{a}_{n+1} / \tilde{a}_{n+3}\right)\right)^{-1}>1 / 3$. It follows from sup $\tilde{a}_{n+1} / \tilde{a}_{n}<4$ and Lemma A-3 that $\eta_{n+2}^{+}<2$. Thus we can take $C=6$. For negative $n$ we have the same result. Thus $N_{n+1}^{-1}$ is uniformly bounded in $n$ and $\lambda$.

Here, we decompose the main part of (44) into

$$
\left|P_{n}\right| \leq\left|\sum_{m=n}^{M} m\left\{(-1)^{m+1} \prod_{i=n+1}^{m} \eta_{i}^{+}\left|b_{m}\right|\right\}\right|+\left|\sum_{m=M+1}^{\infty}(-1)^{m+1} m \prod_{i=n+1}^{m} \eta_{i}^{+}\right| b_{m}|| .
$$

Note that only the second term remains for $n>M$. If we suppose that $\prod_{i=n+1}^{m} \eta_{i}^{+}$and $\left|b_{m}\right|$ are monotone decreasing, then both terms in (45) are estimated by $M\left|b_{n}\right|$. Indeed, Abel's criterion for summability is applicable to the first term. In the second term we can verify that $m \eta_{i}^{+}\left|b_{m}\right|$ is monotone decreasing. Now define $\bar{b}_{m}$ by

$$
\bar{b}_{m} \equiv b_{m} \quad \text { if }\left|\rho_{m}\right| \leq 1, \quad b_{m-1} \quad \text { if }\left|\rho_{m}\right|>1 .
$$

We see, from (33), that the sequence $\left\{\left|\bar{b}_{m}\right|\right\}$ is monotone decreasing. Note that $\eta_{i}^{+}$has a property similar to quasi-monotonicity. So let us use the same notation $S(\lambda)$ also for $\left\{\eta_{i}^{+}\right\}$. We now consider the modification of $B(n, m) \equiv \prod_{i=n+1}^{m} \eta_{i}^{+}$defined by

$$
\bar{B}(n, m) \equiv B(n, m) \quad \text { if } \eta_{m}^{+} \leq 1, \quad B(n, m-1) \quad \text { if } \eta_{m}^{+}>1 .
$$

Then it follows that

$$
\left|\sum_{m=n}^{M}(-1)^{m+1} m \bar{B}(n, m)\right| \bar{b}_{m}|| \leq 2 M\left|b_{n}\right| .
$$

Note that $\bar{B}(n, m)=B(n, m)$ holds if $m>M$.

Now, we need to consider

(46) $\left|\sum_{m=n}^{M}(-1)^{m+1} m B(n, m)\left(\left|b_{m}\right|-\left|\bar{b}_{m}\right|\right)\right|+\left|\sum_{m=n}^{M}(-1)^{m+1} m(B(n, m)-\bar{B}(n, m))\right| \bar{b}_{m}||$.

Here, by virtue of monotonicity of $\left|\bar{b}_{m}\right|$ and $\bar{B}(n, m)$, Abel's criterion for summability can be applied to each term of (46). Since $\left|b_{m}\right|-\left|\bar{b}_{m}\right|=\left(\max \left\{\left|\rho_{m}\right|-1,0\right\}\right)\left|\bar{b}_{m}\right|$, using the notation $S_{m}(\lambda) \equiv\left(\max \left\{\left|\rho_{m}\right|-1,0\right\}\right) \leq S(\lambda)=\max _{m} S_{m}(\lambda)$, we may rewrite the first term of (46) as 
follows.

$$
\begin{aligned}
& \left|\sum_{m=n}^{M}(-1)^{m+1} m B(n, m)\left(\left|b_{m}\right|-\left|\bar{b}_{m}\right|\right)\right| \\
& \quad \leq\left|\bar{b}_{n}\right|\left|\sup _{n \leq k \leq M} \sum_{m=n}^{k}(-1)^{m+1} m B(n, m) S_{m}(\lambda)\right| \\
& \quad \leq\left|\bar{b}_{n}\right|\left|\sup _{k=n, n+1} \sum_{m=n}^{k}(-1)^{m+1} m B(n, m) S_{m}(\lambda)\right|+\left|\bar{b}_{n}\right|\left\{S(\lambda) \sum_{m=n+2}^{M} m B(n, m)\right\} .
\end{aligned}
$$

Let us now recall the argument in the proof of $\left(34^{\prime}\right)$. Then, replacing $\rho_{k}$ by $\eta_{k}^{+}$, we obtain

(48) $\quad B(n, m) \leq(1+S(\lambda)) \bar{B}(n, m), \quad \bar{B}(n, m) \leq \exp \left\{-M^{-4}(2[(m-n) / 2])^{5} / 20\right\}$.

From (48) and $\exp \left\{-M^{-4}(2[(m-n) / 2])^{5} / 20\right\} \leq \exp \left\{-M^{-4}(m-n-1)^{5} / 20\right\}$, we have

$$
\begin{aligned}
& \sum_{m=n+2}^{M} m \bar{B}(n, m)<\int_{n+2}^{M+1} t \exp \left\{-\left(M^{-4}(t-n-2)^{5}\right) / 20\right\} d t \\
= & \int_{0}^{M-n-1} s \exp \left(-M^{-4} s^{5} / 20\right) d s+\int_{0}^{M-n-1}(n+2) \exp \left(-M^{-4} s^{5} / 20\right) d s \\
< & M^{2} \int_{0}^{1} r \exp \left(-M r^{5} / 20\right) d r+(n+2) M \int_{0}^{1} \exp \left(-M r^{5} / 20\right) d r \\
= & \frac{1}{5} M^{2} \int_{0}^{1} y^{-3 / 5} \exp (-M y / 20) d y+\frac{1}{5}(n+2) M \int_{0}^{1} y^{-4 / 5} \exp (-M y / 20) d y \\
= & \frac{1}{5} M^{8 / 5} \int_{0}^{M} x^{-3 / 5} \exp (-x / 20) d x+\frac{1}{5}(n+2) M^{4 / 5} \int_{0}^{M} x^{-4 / 5} \exp (-x / 20) d x \\
< & \left(2^{4} / 5^{3}\right)^{1 / 5} \Gamma(2 / 5) M^{8 / 5}+\left(2^{2} / 5^{4}\right)^{1 / 5} \Gamma(1 / 5) M^{4 / 5}(n+2) \equiv C_{1}^{\prime} M^{8 / 5}+C_{2}^{\prime} M^{4 / 5}(n+2) .
\end{aligned}
$$

Thus the second term of (47) is bounded by

$2\left|\bar{b}_{n}\right|\left\{3(2 c+1) M^{-2 / 3}\right\}\left\{C_{1}^{\prime} M^{8 / 5}+C_{2}^{\prime} M^{4 / 5}(n+2)\right\} \equiv\left|\bar{b}_{n}\right|\left\{C_{1}^{\prime \prime} M^{14 / 15}+C_{2}^{\prime \prime} M^{2 / 15}(n+2)\right\}$.

The first term of (47), which is the sum of terms with different sign, is smaller than $\left|\bar{b}_{n}\right|\left\{2\left(C M^{-2 / 3}\right)\right\}(n+1)$. Hence for some positive constants $C_{1}$ and $C_{2}$ we have

$$
\left|\sum_{m=n}^{M}(-1)^{m+1} m B(n, m)\left(\left|b_{m}\right|-\left|\bar{b}_{m}\right|\right)\right|<\left|\bar{b}_{n}\right|\left\{C_{1} M^{14 / 15}+C_{2} M^{2 / 15}(n+2)\right\} .
$$

As for the second term of (46), we can obtain the same estimate as in (49), that is,

$\left(49^{\prime}\right)\left|\sum_{m=n}^{M}(-1)^{m+1} m(B(n, m)-\bar{B}(n, m))\right| \bar{b}_{m}||<\left|\bar{b}_{n}\right|\left\{C_{1} M^{14 / 15}+C_{2} M^{2 / 15}(n+2)\right\}$, 
since we have $B(n, m)-\bar{B}(n, m) \leq S_{m}(\lambda) \bar{B}(n, m)$. From (45), (45'), (46), (49) and (49') it follows that

$$
\left|\sum_{m=n}^{\infty}(-1)^{m+1} m \prod_{i=n+1}^{m} \eta_{i}^{+}\right| b_{m}||<\left|\bar{b}_{n}\right|\left(c_{1} M+c_{2} M^{2 / 15} n\right), \quad n \geq 2 .
$$

We want to show that the right hand side of (43) is much smaller, by ratio, than the quantity given by (27), for sufficiently large $M$. Since the terms in the right hand side of (43) are estimated in the same way, we only remark on the first term of (43) (see Proposition 6 and Corollary 1). Then, from (44) and $\lim _{M \rightarrow \infty} M^{2} / \lambda=1$, we have

$$
2 \sum_{n=2}^{\infty}\left|\sum_{m=n}^{\infty} \tilde{c}_{m, n}\right|<\sum_{n=2}^{\infty}\left|b_{n}\right|^{2}\left(c M^{-1} n+c^{\prime} M^{-28 / 15} n^{2}\right),
$$

where $c$ and $c^{\prime}$ are some constants. Now we can see that for each finite $n$ the term in (50) is evidently small, when $M$ is large. In order to see (50), we divide $\mathbf{N}$ into two parts $\{n ; 1 \leq$ $\left.n \leq k_{0}\right\}$ and $\left\{n ; n>k_{0}\right\}$, and take $k_{0}=\delta M^{14 / 15}$ with small positive constant $\delta$ which is determined later. Then the terms in (50) for $n \leq k_{0}$ are smaller than the corresponding terms in (27), and for $n>k_{0}$ we obtain the following inequality using (32) with $n^{\prime}=0$ and (35):

$$
\begin{aligned}
& \sum_{n=k_{0}}^{\infty}\left|b_{n}\right|^{2}\left(c M^{-1} n+c^{\prime} M^{-28 / 15} n^{2}\right) \\
& \leq \int_{k_{0}}^{\infty}(1+S(\lambda))^{2} \exp \left(-M^{-4} t^{5} / 10\right)\left(c M^{-1} t+c^{\prime} M^{-28 / 15} t^{2}\right) d t \\
& <(1+S(\lambda))^{2}\left\{c \int_{\delta}^{\infty} s \exp \left(-M^{2 / 3} s^{5} / 10\right) M^{13 / 15} d s\right. \\
& \left.+c^{\prime} \int_{\delta}^{\infty} s^{2} \exp \left(-M^{2 / 3} s^{5} / 10\right) M^{14 / 15} d s\right\},
\end{aligned}
$$

which converges to zero for any fixed positive number $\delta$ as $M$ tends to $\infty$.

Let us now return to the terms $\left|\tilde{c}_{1,1}\right|$ and $\left|\sum_{n=2}^{\infty} \tilde{c}_{m, 1}\right|$. Since they are represented respectively by $\left(N_{2} / \lambda\right)\left|b_{1}\right|^{2}$ and $\left(N_{2} / \lambda\right)\left|b_{1}\right|\left|\sum_{m=2}^{\infty}(-1)^{m+1} m \prod_{i=2+1}^{m} \eta_{i}^{+}\right| b_{m}||$, these values are also much smaller than the value given by (27). Note that (27) contains only the terms for odd $n$. However, we remark that the quasi-monotonicity (35) plays an important role in compensating the even terms. Now note that the first and the second terms in (27) are positive as $M$ tends to $\infty$. Indeed, it is verified by showing that $\lim _{\inf } \rightarrow \infty\left|b_{n}\right|>(25 / 34)^{n}, n<M$, which are derived from the formula

$$
\begin{aligned}
\liminf _{M \rightarrow \infty}\left|\rho_{j}\right| & >\frac{1}{1+\left(a_{j} / a_{j+1}\right)}=\frac{1}{1+f(j)}, \\
f(j) & =\frac{(j+(1 / j))^{2}}{(j+2+(1 /(j+2)))^{2}}, \quad j \geq 1 .
\end{aligned}
$$

The inequality (51) follows from (35) and $\left(1+a_{j} a_{j+1}+\left(a_{j} / a_{j+2}\right)\right)^{-1}<\rho_{j} \rho_{j+1}, \quad j \geq 1$. Here we see that the $\{f(j)\}_{j=2}^{\infty}$ is a monotone increasing sequence satisfying $f(2)=10^{2} / 17^{2}$ 
and $\lim _{j \rightarrow \infty} f(j)=1$. Since $1>f(1)=3^{2} / 5^{2}>10^{2} / 17^{2}$, we have $\liminf _{M \rightarrow \infty}\left|\rho_{j}\right| \geq$ $\left(1+\left(10^{2} / 17^{2}\right)\right)^{-1}$ for $j \geq 1$. Thus we can show that $b_{1}^{2} / \lambda$ in $\bar{D}_{2}^{(k)}$ is much smaller than the first term of $\bar{D}_{1}^{(k)}$ for large $\lambda$. To see this, it suffices to note $\left(\left(3^{2}-1\right) / 3^{2}\right) b_{3}^{2}=\left(\left(3^{2}-\right.\right.$ 1) $\left./ 3^{2}\right) b_{1}^{2}\left(\rho_{2} \rho_{3}\right)^{2}$ and $\rho_{2} \rho_{3} \geq\left(1+a_{2} a_{3}+\left(a_{2} / a_{4}\right)\right)^{-1}=\left(1+a_{1} a_{2}+(10 / 17)^{2}\right)^{-1}$. Therefore we obtain that $\bar{D}_{1}^{(k)}>\left|\bar{D}_{2}^{(k)}\right|$ if $\beta=k \alpha<1$ is sufficiently close to 1 . Hence in this case we have

$$
D_{1}^{(k)}>\left|D_{2}^{(k)}\right|
$$

Thus the proof of Theorem 2 is complete.

Now we note that, as remarked before for sufficiently small $\beta>0$, we obtain (52) from the representations $D_{1}^{(k)}$ and $D_{2}^{(k)}$. It is desirable to verify (52) for closed interval of $\beta$ in $(0,1)$, that is, for $\lambda$ belonging to a compact region $\left[\sqrt{2}+\delta^{\prime}, 1 / \delta^{\prime}\right]$ with small positive $\delta^{\prime}$, using the explicit formula for $D_{1}^{(k)}+D_{2}^{(k)}$ given rigorously. In this region, since all the terms are uniformly bounded and all the series are rapidly and absolutely convergent, $D_{1}^{(k)}$ and $D_{2}^{(k)}$ are uniformly continuous. So the numerical computation is possible by choosing finite numbers of points. Incidentally, we remark that $\lambda$ is a solution of (9) which is resolved by a successive approximation method for the implicit function.

APPENDIX. Continued fractions are used by many authors to analyze delicate problems (see for example [9]). For the convenience of readers we collect some basic results on which our arguments are based.

Proposition A-1. The problem (1), (2) and (3) is equivalent to the problem (1'), $\left(2^{\prime}\right)$ and $\left(3^{\prime}\right)$.

PROOF. The first equation in $\left(1^{\prime}\right)$ is verified by taking the rotation and the divergence of (1). Conversely, from (1') by the same process we have (1) operated by $\Delta$. Note that the inverse operator of $\Delta$ exists on the subspace consisting of functions with mean value zero. As for the periodicity, for example, $\psi(x, y)=\psi(x, y+2 \pi)$, we note the relations

$$
\begin{aligned}
\psi(x, y+2 \pi)-\psi(x, y) & =\left(\int_{(x, y)}^{(s, y)}+\int_{(s, y)}^{(s, y+2 \pi)}+\int_{(s, y+2 \pi)}^{(x, y+2 \pi)}\right) u d y-v d x \\
& =\int_{(s, y)}^{(s, y+2 \pi)} u d y-v d x=\int_{(s, y)}^{(s, y+2 \pi)} u d y, \quad s \in(0,2 \pi / \alpha), \\
\int_{-\pi / \alpha}^{\pi / \alpha} & \left(\int_{(s, y)}^{(s, y+2 \pi)} u d y\right) d s=\iint_{D} u d x d y=0 .
\end{aligned}
$$

Hence it holds that $\psi(x, y+2 \pi)-\psi(x, y)=0$, and similarly follows $\psi(x+2 \pi / \alpha, y)-$ $\psi(x, y)=0$. The mean value of $v u_{y}=\left(-\psi_{x}\right)\left(\psi_{y y}\right)$, for example, is equal to that of $\psi_{x y} \psi_{y}=(1 / 2)\left(\partial \psi_{y}^{2} / \partial x\right)$, which is zero by the periodicity of $\psi$. 
LEMMA A-1. Suppose that a sequence of positive numbers $\left\{a_{k}\right\}_{k=1}^{\infty}$ satisfies the relation $0<\inf _{i \in \mathbf{N}} a_{i} a_{i+1}=1 / p$, and put

$$
\rho_{1, n} \equiv \frac{1}{a_{1}}+\frac{1}{a_{2}}+\cdots+\frac{1}{a_{n}}, \quad n \in \mathbf{N} .
$$

Then the limit $\rho_{1} \equiv \lim _{n \rightarrow \infty} \rho_{1, n}$ exists and satisfies

$$
\left|\rho_{1, n}-\rho_{1}\right| \leq \max \left\{1,1 / a_{1}^{2}\right\} p^{1 / 2}(1+(1 / p))^{-1}(p /(1+p))^{n-2}, \quad 2<n, \quad n \in \mathbf{N} .
$$

Proof. Put

$$
\rho_{i, n} \equiv \frac{1}{a_{i}}+\frac{1}{a_{i+1}}+\cdots+\frac{1}{a_{n}}, \quad i \leq n .
$$

For fixed $i \in \mathbf{N}$ and any $k>i$, we can verify $\rho_{i, i}>\rho_{i, k}, \rho_{i, i+1}<\rho_{i, k+1}, \ldots$, and

$$
\rho_{i, i+2 j}>\rho_{i, k+2 j}, \quad \rho_{i, i+2 j+1}<\rho_{i, k+2 j+1}, \quad i<k, \quad j \in \mathbf{N} .
$$

From this inequality we have

$$
\rho_{i, i+2(j-1)}>\rho_{i, i+2 j}, \quad \rho_{i, i+2 j-1}<\rho_{i, i+2 j+1}, \quad \rho_{i, i+2(j-1)}>\rho_{i, i+2 j-1}, \quad j \in \mathbf{N} .
$$

Note that $\left|\rho_{i, n}-\rho_{i, n+1}\right|$ is monotone decreasing in $n$. Therefore the limit $\rho_{i} \equiv \lim _{n \rightarrow \infty} \rho_{i, n}$ exists if and only if $\lim _{n \rightarrow \infty}\left|\rho_{i, n}-\rho_{i, n+1}\right|=0$, which means that $\left\{\rho_{i, n}\right\}_{n=i+1}^{\infty}$ is a Cauchy sequence in this case. On the other hand, by definition, it holds that, for $j=1,2$ and $m>2$,

$$
\left|\rho_{j, m}-\rho_{j, m+1}\right|=\left|\rho_{j, m} \rho_{j, m+1}\right|\left|\rho_{j+1, m}-\rho_{j+1, m+1}\right|=\prod_{i=j}^{m-1}\left|\rho_{i, m} \rho_{i, m+1}\right|\left|\rho_{m, m}-\rho_{m, m+1}\right| .
$$

Here note that for $m \in \mathbf{N}$, we have

$$
\begin{aligned}
\left|\rho_{m, m}-\rho_{m, m+1}\right| & =\left|\left(a_{m}\right)^{-1}-\left(a_{m}+\left(1 / a_{m+1}\right)\right)^{-1}\right| \\
& =\left(a_{m}\left(1+a_{m} a_{m+1}\right)\right)^{-1}<\left(a_{m}(1+(1 / p))\right)^{-1} .
\end{aligned}
$$

Since the assumption $0<\inf _{m \in \mathbf{N}} a_{m} a_{m+1}=1 / p$ yields that $\sup _{m \in \mathbf{N}} \min \left\{1 / a_{m}, 1 / a_{m+1}\right\} \leq$ $p^{1 / 2}$, we have $\left|\rho_{m, m}-\rho_{m, m+1}\right|<p^{1 / 2} /(1+(1 / p))$ for $m=n$ or $m=n-1$, which we denote by $m=m(n)$. On the other hand, since $\rho_{i, k}=1 /\left(a_{i}+\rho_{i+1, k}\right)$ for any $i<k$, we have $\left|\rho_{i, k} \rho_{i+1, k}\right|=1-a_{i} \rho_{i, k}<1-a_{i}\left(a_{i}+\left(1 / a_{i+1}\right)\right)^{-1} \leq 1-(1+p)^{-1}=p(1+p)^{-1}, \quad i<k$.

Hence, for $j$ satisfying $(m-j) / 2 \in \mathbf{N}$ it follows that

$$
\prod_{i=j}^{m-1}\left|\rho_{i, m} \rho_{i, m+1}\right|=\prod_{k=0}^{(m-j) / 2}\left|\rho_{j+2 k, m} \rho_{j+2 k+1, m}\right|\left|\rho_{j+2 k, m+1} \rho_{j+2 k+1, m+1}\right| \leq(p /(1+p))^{m-j} .
$$

If $m-1$ is even, then we have

$$
\left|\rho_{1, n}-\rho_{1, n+1}\right| \leq\left|\rho_{1, m}-\rho_{1, m+1}\right| \leq p^{1 / 2}(1+(1 / p))^{-1}(p /(1+p))^{m-1} .
$$


If $m-1$ is odd, then we have

$$
\begin{aligned}
\left|\rho_{1, n}-\rho_{1, n+1}\right| & \leq\left|\left(a_{1}+\rho_{2, n}\right)^{-1}-\left(a_{1}+\rho_{2, n+1}\right)^{-1}\right|<\frac{1}{a_{1}^{2}}\left|\rho_{2, n}-\rho_{2, n+1}\right| \\
& \leq \frac{1}{a_{1}^{2}}\left|\rho_{2, m}-\rho_{2, m+1}\right| \leq a_{1}^{-2} p^{1 / 2}(1+(1 / p))^{-1}(p /(1+p))^{m-2} .
\end{aligned}
$$

In general, we have that $\left|\rho_{1, n}-\rho_{1, n+1}\right| \leq \max \left\{1,1 / a_{1}^{2}\right\} p^{1 / 2} /(1+(1 / p))^{-1}\{p /(1+p)\}^{n-1}$.

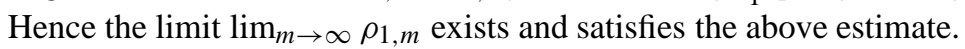

Proposition A-2. If we take $\rho_{1}$ different from $\rho_{1}^{+}$, then the solution $\rho_{n}$, $n=2,3, \ldots$, of (7) satisfies $\lim _{n \rightarrow+\infty}\left|\rho_{n}\right|=\infty$.

Proof. Since $\lim _{n \rightarrow+\infty} \rho_{n}^{+}=0$, there exists $n_{0} \in \mathbf{N}$ such that $\left|\rho_{n}^{+}\right|<1 / 3$ holds for all $n \geq n_{0}$. Now suppose that $\left\{\rho_{n}\right\}$ satisfies the system of equations in (7) with given $\rho_{1} \neq \rho_{1}^{+}$. For $n \geq n_{0}$, it holds that

$$
\begin{aligned}
\left|\rho_{n}-\rho_{n}^{+}\right| & =\left|\left(a_{n-1}+\left(1 / \rho_{n-1}\right)\right)-\left(a_{n-1}+\left(1 / \rho_{n-1}^{+}\right)\right)\right|=\left|\rho_{n-1}^{+}-\rho_{n-1}\right| /\left|\rho_{n-1} \rho_{n-1}^{+}\right| \\
& =\left|\rho_{n_{0}}^{+}-\rho_{n_{0}}\right| /\left|\rho_{n-1} \rho_{n-2} \cdots \rho_{n_{0}} \rho_{n-1}^{+} \rho_{n-2}^{+} \cdots \rho_{n_{0}}^{+}\right| .
\end{aligned}
$$

Since $\left|\rho_{n-1} \cdots \rho_{n_{0}}\right|\left|\rho_{n}-\rho_{n}^{+}\right|>3^{n-n_{0}}\left|\rho_{n_{0}}^{+}-\rho_{n_{0}}\right|$ for any $n \geq n_{0}$, we see that $\lim _{\sup _{n \rightarrow \infty}}\left|\rho_{n}\right| \geq 3$. On the other hand, from $\rho_{n}-\rho_{n}^{+}=1 / \rho_{n-1}-1 / \rho_{n-1}^{+}$, we have

$$
\left|\rho_{n}\right| \geq\left|1 / \rho_{n-1}^{+}\right|-\left\{\left|\rho_{n}^{+}\right|+\left|1 / \rho_{n-1}\right|\right\} .
$$

If $\left|\rho_{n-1}\right|>2$, then $\left|\rho_{n}\right|>2+1 / 6$ for $n \geq n_{0}+1$. Hence $\lim \sup _{n \rightarrow \infty}\left|\rho_{n}\right| \geq 3$ implies that $\lim \sup _{n \rightarrow \infty}\left|1 / \rho_{n}\right|<2$. Thus $\lim _{n \rightarrow \infty} \rho_{n}^{+}=0$ yields $\lim _{n \rightarrow+\infty}\left|\rho_{n}\right|=\infty$.

LEMMA A-2. Suppose that each $a_{k}(s)$ is a continuous function of a parameter $s \in$ $\left(s_{0}, s_{1}\right)$ and satisfies the condition in Lemma A-1 uniformly in $s$. Then $\rho_{n}(s)$ defined in Lemma A-1 is a continuous function of $s$. Moreover, assume that each $a_{k}(s)$ is a continuously differentiable function satisfying $d a_{2 k-1} / d s(s)>0$ for $k \in \mathbf{N}$ and $d a_{2 k} / d s(s)<0$ for $k \in \mathbf{N}$. Then $\rho_{1}(s)$ is a monotone decreasing function of $s$.

PROOF. As $\rho_{1}(s)$ is the uniform limit of continuous functions $\left\{\rho_{1, m}(s)\right\}, \rho_{1}(s)$ is continuous in $s$. Suppose $s_{1}<s_{2}$. Then we have

$$
\rho_{1,2 j+1}\left(s_{1}\right)-\rho_{1,2 j}\left(s_{2}\right)=\left(\rho_{1,2 j+1}\left(s_{1}\right)-\rho_{1,2 j+1}\left(s_{2}\right)\right)+\left(\rho_{1,2 j+1}\left(s_{2}\right)-\rho_{1,2 j}\left(s_{2}\right)\right) .
$$

Note that the second term converges to 0 as $j$ tends to zero. Remark that we can show

$$
\frac{d \rho_{1,2 j+1}}{d s}(s)<-\frac{a_{1}^{\prime}(s)}{\left(a_{1}(s)+\left(1 / a_{2}(s)\right)\right)^{2}}<0
$$

for any $j \in \mathbf{N}$ and any $s$. Hence it follows that $\rho_{1}\left(s_{1}\right)-\rho_{1}\left(s_{2}\right)<0$.

LemmA A-3. Assume that a positive sequence $\left\{a_{n}\right\}_{n=1}^{\infty}$ satisfies $\sup _{1 \leq n} a_{n+1} / a_{n}<$ $p^{2}$. Put $\rho_{n}=\frac{1}{a_{n}}+\frac{1}{a_{n+1}}+\cdots+\frac{1}{a_{k}}+\cdots, n=1,2, \ldots$. If $\lim _{n \rightarrow \infty} \rho_{n}=0$, then $\sup _{1 \leq n} \rho_{n}<p$. 
ProOF. We see that $\rho_{k}=\rho_{k}(\beta, \lambda) \geq p$ implies that $\left\{\rho_{k+2 j}\right\}_{j=0}^{\infty}$ is an increasing sequence. Indeed, $\rho_{k}=1 /\left(a_{k}+\rho_{k+1}\right)$ and $\rho_{k+1}=1 /\left(a_{k+1}+\rho_{k+2}\right)$ yield that $\rho_{k+2} / \rho_{k}=\{1-$ $\left.a_{k} \rho_{k}\left(a_{k+1} \rho_{k+1} / a_{k} \rho_{k}\right)\right\} /\left(1-a_{k} \rho_{k}\right)$, where $\left(a_{k+1} \rho_{k+1} / a_{k} \rho_{k}\right)<\left(a_{k+1} / a_{k}\right)\left(1 /\left(\rho_{k}\right)^{2}\right)$ follows from $\rho_{k} \rho_{k+1}=1-a_{k} \rho_{k}<1$. Therefore, $\rho_{k} \geq p$ implies that $\rho_{k}<\rho_{k+2}$. Similarly, we have that $\rho_{k+2}<\rho_{k+4}<\cdots$, which contradicts $\lim _{n \rightarrow \infty} \rho_{n}=0$.

Proofs of (i) AND (ii) IN SUbSECtION 1.3. First note that $a_{n}>0, n=1,2, \ldots$, for $\beta>1$ and $a_{0}=\infty$ for $\beta=1$. Therefore (i) follows. To verify (ii) we note

$$
g(\beta, \lambda)=\lambda \beta^{-3}\left(\rho_{1}^{-}(\beta, \lambda)-\rho_{1}^{+}(\beta, \lambda)\right)=\tilde{a}_{0}+2\left(\frac{1}{\tilde{a}_{1}}+\frac{1}{\tilde{a}_{2}}+\cdots\right)=0,
$$

where

$$
\left\{\begin{array}{l}
\tilde{a}_{n}=2\left(\beta^{2}+n^{2}\right)^{2} /\left(\beta^{4}\left(\beta^{2}+n^{2}-1\right)\right), \quad n=2 k, k=0,1,2, \ldots, \\
\tilde{a}_{n}=2\left(\beta^{2}+n^{2}\right)^{2} \beta^{2} /\left(\lambda^{2}\left(\beta^{2}+n^{2}-1\right)\right), \quad n=2 k+1, k=0,1,2, \ldots
\end{array}\right.
$$

Then, by considering logarithmic derivatives, we can show $\partial \tilde{a}_{n} / \partial \beta<0$ for all even $n$ and $\partial \tilde{a}_{n} / \partial \beta>0$ for all odd $n$. This implies that $g(\beta, \lambda)$ is a decreasing function in $\beta$, namely, $g\left(\beta_{1}, \lambda\right)>g\left(\beta_{2}, \lambda\right)$ holds for $0<\beta_{1}<\beta_{2}<1$ (see Lemma A-2). Similarly, we can show that $g(\beta, \lambda)$ is an increasing function with respect to $\lambda$.

Note that we have $\lim _{\lambda \rightarrow 0} g(\beta, \lambda)=\tilde{a}_{0}<0$ and $\lim _{\lambda \rightarrow \infty} g(\beta, \lambda)=\infty$. Hence the solution $\lambda=\lambda(\beta)$ of $g(\beta, \lambda)=0$ exists uniquely for $\beta \in(0,1)$. Moreover, $\lambda(\beta)$ is a monotone increasing function. Indeed, $0<\beta_{1}<\beta_{2}<1$ yields that $0=g\left(\beta_{1}, \lambda\left(\beta_{1}\right)\right)>$ $g\left(\beta_{2}, \lambda\left(\beta_{1}\right)\right)$ and $g\left(\beta_{2}, \lambda\left(\beta_{1}\right)\right)<g\left(\beta_{2}, \lambda\left(\beta_{2}\right)\right)=0$, which implies that $\lambda\left(\beta_{1}\right)<\lambda\left(\beta_{2}\right)$. Since $0 \equiv \lim _{\beta \rightarrow 0} g(\beta, \lambda(\beta))=\lim _{\beta \rightarrow 0}\left(\tilde{a}_{0}+\left(1 / \tilde{a}_{1}\right)\right)=-2+\left(\lim _{\beta \rightarrow 0} \lambda(\beta)\right)^{2}$, we have $\lim _{\beta \rightarrow 0} \lambda(\beta)=\sqrt{2} .1 / 6<\rho_{1}^{+}<2$ follows from (iii) and $1 / 3<\rho_{1}^{+} \rho_{2}^{+}$, Therefore, we have (ii), since $\left|a_{0}\right|=2\left|\rho_{1}^{+}\right|$.

Proof of Theorem 4. Here we give the proof of Theorem 4, compared with Proposition 6. The $(m, m)$ element of $\mathbf{D}^{-1}$ is equal to $N_{m+1}^{-1}$. By an argument similar to that for Proposition 6, we can verify that $\left\{N_{m+1}^{-1}\right\}$ is bounded in $m \in \mathbf{Z}$. Other $(m, n)$ elements of $\mathbf{D}^{-1}$ are also described by $N_{m+1}^{-1}$ and $\rho_{i}^{+}, i=m+1, \ldots, n$, if $n>m$. If $m>n$, we use $N_{m+1}^{-1}$ and $1 / \rho_{j}^{-}, j=n+1, \ldots, m$. Therefore, since $\rho_{i}^{+}, i=m+1, \ldots, n$ and $1 / \rho_{j}^{-}$, $j=n+1, \ldots, m$ are bounded, we have the uniform estimate stated in Theorem 4 , since $\rho_{i}^{+} \rho_{i+1}^{+}<1$, etc.

Acknowledgment. The authors express their gratitude to Professors H. Okamoto, K. Masuda, referees and editors for continuous encouragement and invaluable suggestions.

\section{REFERENCES}

[1] L. D. Meshalkin And Y. G. Sinai, Investigation of the stability of a stationary solution of a system of equations for the plane movement of an incompressible viscous liquid, J. Appl. Math. Mech. 25 (1961), 1700-1705.

[2 ] V. I. IUDOVICH, Example of the generation of a secondary stationary or periodic flow when there is loss of stability of the laminar flow of a viscous incompressible fluid, J. Appl. Math. Mech. 29 (1965), 527-544. 
[ 3 ] M. G. Crandall AND P. H. Rabinowitz, Bifurcation from simple eigenvalues, J. Funct. Anal. 17 (1971), 321-340.

[ 4 ] А. М. Овикноv, Kolmogorov flow and laboratory simulation of it, Russian Math. Surveys 38 (1983), $113-126$.

[ 5 ] H. Окамото AND M. SHŌJI, Bifurcation diagrams in Kolmogorov's problem of viscous incompressible fluid on 2-D flat tori, J. J. Indust. Appl. Math. 10 (1993), 191-218.

[ 6 ] M. YAmADA, Nonlinear stability theory of spatially periodic parallel flows, J. Phys. Soc. Japan 55 (1986), 3073-3079.

[ 7 ] K. MASUDA, Nonlinear mathematics, Asakura, Tokyo (in Japanese), 1986.

[ 8 ] V. X. LIU, An example of instability for the Navier-Stokes equations on the 2-dimensional torus, Comm. Partial Differential Equations 17 (1992), 1995-2012.

[ 9 ] Y. OGURA AND S. SHIMAKURA, Stationary solutions and their stability for Kimura's diffusion model with intergroup selection, J. Math. Kyoto. Univ. 27 (1987), 305-347.

DEPARTMENT OF MATHEMATICS

FACULTY OF SCIENCE AND TECHNOLOGY

SCIENCE UNIVERSITY OF TOKYO

2641 NODA, CHIBA 278-8510

JAPAN

E-mail address:mami@ma.noda.sut.ac.jp
DEPARTMENT OF MATHEMATICS

FACULTY OF SCIENCE

NARA WOMEN'S UNIVERSITY

KITAUOYA-NISHIMATCHI, NARA 630-8506

JAPAN

E-mail address: sadao@cc.nara-wu.ac.jp 